ESAIM: M2AN 48 (2013) 27-52

DOI: $10.1051 / \mathrm{m} 2 \mathrm{an} / 2013093$
ESAIM: Mathematical Modelling and Numerical Analysis

www.esaim-m2an.org

\title{
MULTISCALE MODELLING OF SOUND PROPAGATION THROUGH THE LUNG PARENCHYMA*
}

\author{
Paul Cazeaux ${ }^{1,2}$ and Jan S. Hesthaven ${ }^{3}$
}

\begin{abstract}
In this paper we develop and study numerically a model to describe some aspects of sound propagation in the human lung, considered as a deformable and viscoelastic porous medium (the parenchyma) with millions of alveoli filled with air. Transmission of sound through the lung above $1 \mathrm{kHz}$ is known to be highly frequency-dependent. We pursue the key idea that the viscoelastic parenchyma structure is highly heterogeneous on the small scale $\varepsilon$ and use two-scale homogenization techniques to derive effective acoustic equations for asymptotically small $\varepsilon$. This process turns out to introduce new memory effects. The effective material parameters are determined from the solution of frequencydependent micro-structure cell problems. We propose a numerical approach to investigate the sound propagation in the homogenized parenchyma using a Discontinuous Galerkin formulation. Numerical examples are presented.
\end{abstract}

Mathematics Subject Classification. 93A30, 35B27, 35B40, 74D05, 65M60.

Received September 27, 2012. Revised April 23, 2013.

Published online November 15, 2013.

Understanding the acoustic properties of the lungs is of great interest in a range of medical domains, from the diagnostic of pathologies such as bronchitis and asthma to recently introduced imaging methods of breathing sounds. Medical doctors have been commonly using the analysis of lung sounds for the diagnostic of pathologies, mainly relying on the stethoscope, and have a good empirical understanding of the relationship between the characteristics of lung sounds and underlying pathologies. However, far less is known about the detailed physical mechanisms of sound generation and transmission in the respiratory system. More advanced models could also explain lung contusions due to a blunt, high-velocity impact to the chest as well as exposure to explosions or, for small mammals, to medical ultrasound.

There is extensive experimental data, both in vitro and in vivo, and a relatively good theoretical understanding of the propagation through lung tissue of relatively low-frequency sound, in the audible range. In the frequency band between roughly a few $\mathrm{Hz}$ to $1 \mathrm{kHz}$, the two main components of the lung tissue, air and connective tissue, are acoustically strongly coupled and the resulting mixture behaves like a homogeneous material with high

Keywords and phrases. Mathematical modeling, periodic homogenization, viscoelastic media, fluid-structure interaction, Discontinuous Galerkin methods.

* This work was partially funded by the ANR-08-JCJC-013-01 (M3RS) project headed by C. Grandmont.

1 Université Pierre et Marie Curie-Paris 6, UMR 7598, Laboratoire J.-L. Lions, 75005 Paris, France

2 Inria Projet REO, Rocquencourt, BP 105, 78153 Le Chesnay Cedex, France. cazeaux@ann.jussieu.fr

3 Division of Applied Mathematics, Brown University, 182 George Street, Providence, RI 02912, USA. Jan_Hesthaven@brown.edu 
density of the tissue and high compressibility of the air filling. Acting as a highly dense gas, the tissue produces a very low speed of sound of less than $50 \mathrm{~m} / \mathrm{s}$. As frequency increases above $1 \mathrm{kHz}$, the wavelength approaches the size of the air pockets embedded in the lungs' tissue, called alveoli, and the absorption increases strongly such that no signal in the audible range is detectable through the human lungs above a frequency of .5-1.5 kHz. Note that this low-pass behavior is unique among human organs. By contrast, the typical speed of sound or ultrasound in soft tissue is normally much higher, around $1500 \mathrm{~m} / \mathrm{s}$, comparable to the speed of sound in water. For a review of experimental and theoretical studies on the subject, we refer to [29].

On the contrary, there are very few studies on the propagation of ultrasound through the lung tissue between $10 \mathrm{kHz}$ and $10 \mathrm{Mhz}$, see $v . g$. [9]. Recently published studies indicate that the propagation properties of ultrasound through the lung are far from understood. In particular, it seems that the effective behavior for in vivo lung tissue is very different from the prohibitively high absorption previously measured at all ultrasound frequencies in in vitro lung samples. According to [32], low-frequency ultrasound may permeate the human lungs in situ for frequencies above $10 \mathrm{kHz}$, and propagation properties are highly dependent on the state of inflation of the lung, or on obstructive pathologies in the patients' lungs. An effective speed of sound above $1000 \mathrm{~m} / \mathrm{s}$ was computed for these new ultrasound recordings, highlighting the difference in behavior with the low-frequency models developed in v.g. [30]. Further development of theoretical models, possibly based on more detailed tissue mechanics, could lead to an increased understanding of these experimental observations and a better modeling of the lungs' acoustical properties.

Elastic fibers are the basic constituent of the connective tissue forming the alveolar wall. At small strain, they exhibit a predominantly elastic behavior with relatively small viscous losses. The remaining material consists of blood vessels, cells and a hydrophilic gel [37]. The mechanical properties of composite tissue depend on the relative amounts of their constituents, but also on their geometrical structure [11]. In the lungs, the elastic fibers are arranged as a three-dimensional network.

In previous attempts to derive a law at the macroscopic scale fitting experimental results, the alveolar wall was modeled as an homogeneous elastic or viscoelastic medium $[2,28,35]$. Here, we change this point of view and try to investigate, theoretically and numerically, some macroscopic effects of the heterogeneity of the alveolar material at the micro-scale. Indeed, the previous discussion suggests that we model the lungs as a foamy material with microstructure, consisting of air bubbles separated by a heterogeneous viscoelastic medium which contains elastic fibers mixed with a viscous gel.

We make some simplifying modeling assumptions. First, motivated by the space repetition of the alveoli, we assume that the parenchyma consists of a periodic arrangement of pores with a small period $\varepsilon>0$. We furthermore assume that the heterogeneous matrix constituting the connective tissue obeys the law of linearized viscoelasticity, and interacts with closed pockets filled with a compressible perfect gas modeling the air. Note that this assumes that air does not communicate freely between neighbouring alveoli. This common modeling assumption $[13,30]$ has been validated by experimental studies $[7,21]$ for frequencies above a few hundred hertz.

Once the model is set up, we employ a two-scale homogenization technique $[1,27]$ to derive effective acoustic equations for the composite tissue of the lungs by performing an asymptotic study as $\varepsilon$ goes to zero. In this limit, we obtain equations describing a homogeneous viscoelastic medium without microstructure. The effective coefficients describing this homogenized medium are recovered from the given periodic micro-scale structure. Note that the homogenization of a similar model, in the static case, was considered in [2]. As is well-known [40], the homogenization of heterogeneous viscoelastic composites, by the interaction of temporal and spatial variations of the coefficients in the differential equations of the model, gives rise to new memory effects. Even when the original models present only instantaneous memories, long-term memories are induced during the homogenization process $[33,39]$.

Finally, we face the problem of computing numerical simulations based on our homogenized description of the parenchyma. The appearance of the new memory effects by homogenization as a convolution term makes the equation hard to use directly. It is standard [36] to modify the model so that the the convolution is replaced by additional differential equations. We achieve this by approximating the viscoelastic behavior of the homogenized medium by a generalized Maxwell model with $n$ relaxation frequencies [3] and then propose 
an efficient strategy based on a Discontinuous Galerkin method [16], which is a finite element method with discontinuities at the interfaces of the elements. Discontinuous Galerkin methods have been widely studied recently, as they combine many advantages, such as adaptativity to complex geometries, high parallelizability and high-order approximation. We present here a high-order Discontinuous Galerkin discretization adapted to the integration of the viscoelastic system in the time domain, with an implicit-explicit time-stepping scheme designed to minimize the computational cost of the method.

The paper is organized as follows. In Section 1, we describe the parenchyma model and we study theoretically this fluid-structure interaction system. In particular, we sketch the homogenization procedure, which consists of deriving the homogenized limit as $\varepsilon$ goes to zero of the model (1.5) by the two-scale convergence framework, and we obtain a macroscopic description involving new memory effects, which depend on the micro-structure of the material.

Then, in Section 2, we propose a numerical method designed to efficiently solve the difficult viscoelastic problem obtained by homogenization. We introduce an efficient approximation of the memory terms by the use of Prony series and detail the discretization of the equations system by the Discontinuous Galerkin method. Finally, in Section 3, we illustrate this study by some numerical results in two dimensions and discuss its relevance to observations.

\section{The VISCOELASTiC HOMOGEnIZED MODEL}

\subsection{The microscale model}

Consider a system composed of a porous, inhomogeneous, incompressible viscoelastic frame perforated by gaseous bubbles. Let the tissue sample be contained in $\Omega$, a bounded domain in $\mathbb{R}^{d}, d=2$ or 3 , which is supposed to be stress-free as the reference configuration of the composite. Assume that $\varepsilon$ denotes a characteristic length of the pore size. We assume that the microscopic and macroscopic scale are well separated, meaning that the parameter $\varepsilon$ is small. To deal with the two different length scales associated with variations of the variables at the micro- and macro-scale, we introduce, in addition to the global position vector $\mathbf{x}$, a local position vector $\mathbf{y}$ in a stretched coordinate system, which is related to $\mathbf{x}$ by

$$
\mathbf{y}=\mathbf{x} / \varepsilon .
$$

For example, if $E_{\varepsilon}(\mathbf{x})=E(\mathbf{x} / \varepsilon)$ was to describe a coefficient appearing in the constitutive equations, then we could suppose that it depends on the fast variable $\mathbf{y}$ as a constant in some component of the material and a different constant in another part of the material to describe heterogeneity of the tissue at the micro-scale.

\subsubsection{The microscale geometry}

Let us be more precise in our description of the material geometry. We restrict our attention to a periodic setting. Let $\mathbf{Z}$ be a reference periodic array in $\mathbb{R}^{d}$ with a reference periodicity cell $\mathcal{Y}$, normalized so that $|\mathcal{Y}|=1$. The bounded domain $\Omega \subset \mathbb{R}^{d}$ is filled with periodically distributed gaseous bubbles. The viscoelastic matrix is assumed connected while the gaseous bubbles are isolated. The reference cell $\mathcal{Y}$ is therefore divided into two parts: an open simply connected bubble $\mathcal{Y}_{F}, \overline{\mathcal{Y}_{F}} \subset \mathcal{Y}$ having a smooth boundary $\Gamma$ and its complement $\mathcal{Y}_{S}=\mathcal{Y} \backslash \overline{\mathcal{Y}_{F}}$ representing the viscoelastic skeleton, see Figure 1. Hence, the viscoelastic material occupies the domain $\Omega_{\varepsilon}$ obtained by $\varepsilon$-periodicity after excluding the bubbles strictly included in $\Omega$ :

$$
\Omega_{\varepsilon}=\bigcup_{\mathbf{k} \in \mathbf{Z}_{\varepsilon}^{\Omega}} \varepsilon\left(\mathcal{Y}_{S}+\mathbf{k}\right), \quad \mathbf{Z}_{\varepsilon}^{\Omega}=\left\{\mathbf{k} \in \mathbf{Z}: \quad \varepsilon\left(\mathcal{Y}_{F}+\mathbf{k}\right) \subset \Omega\right\}
$$




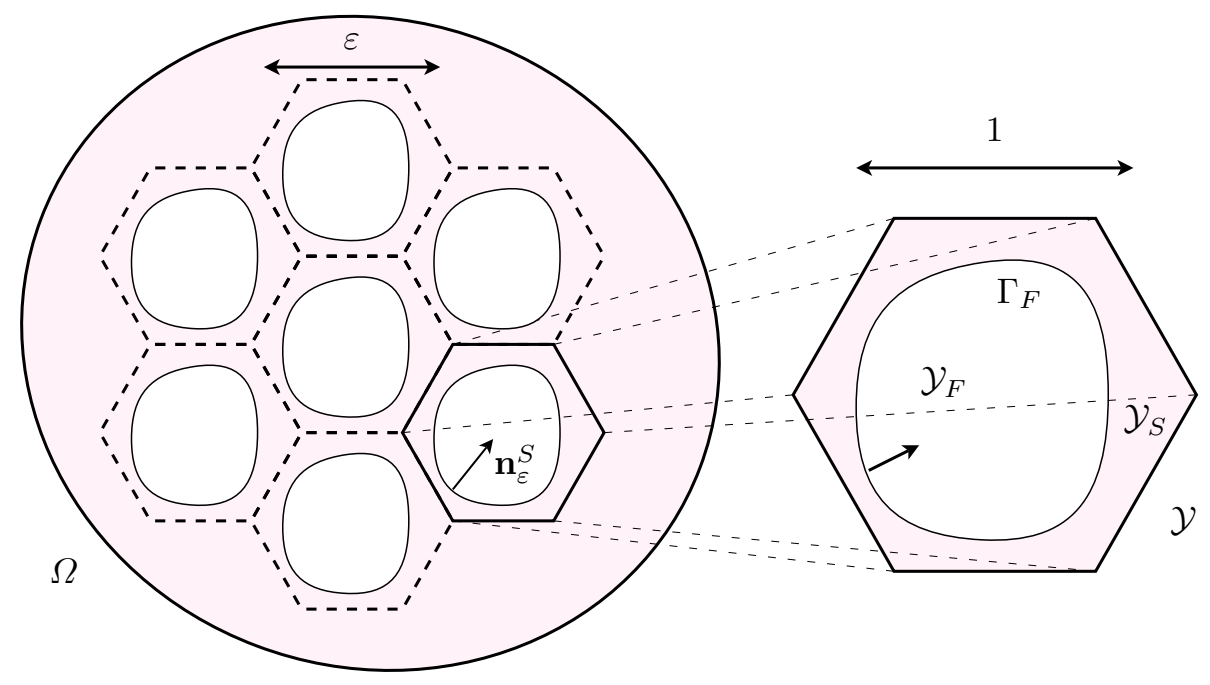

FiguRE 1. Domain $\Omega_{\varepsilon}$ and unit fluid-structure cell $\mathcal{Y}$.

\subsubsection{The microscale model equations}

We will consider motions of the medium small enough to be governed by linearized equations and denote the gaseous bubbles as $\mathcal{B}_{\varepsilon}^{\mathbf{k}}=\varepsilon\left(\mathcal{Y}_{F}+\mathbf{k}\right)$ for $\mathbf{k} \in \mathbf{Z}_{\varepsilon}^{\Omega}$. Suppose that the closed gas bubbles behave like a perfect gas experiencing adiabatic compression, so the relation between volume $V_{\varepsilon}^{\mathbf{k}}(t)$ and pressure $p_{\varepsilon}^{\mathbf{k}}$ of the $\mathbf{k}$-bubble at time $t$ is given by the law

$$
p_{\varepsilon}^{\mathbf{k}}(t)\left(V_{\varepsilon}^{\mathbf{k}}(t)\right)^{\gamma}=\text { Cst. }
$$

where $\gamma$ is the specific heat ratio for the gas (about 1.4 for air). Let $\mathbf{u}_{\varepsilon}$ be the (small) displacement field of the elastic media. Linearizing around an equilibrum state of atmospheric pressure $p_{a}$ and volume $\left|\mathcal{B}_{\varepsilon}^{\mathbf{k}}\right|$, the equation governing the pressure inside the bubbles is [2]:

$$
p_{\varepsilon}^{\mathbf{k}}-p_{a} \approx \frac{\gamma p_{a}}{\left|\mathcal{B}_{\varepsilon}^{\mathbf{k}}\right|} \int_{\partial \mathcal{B}_{\varepsilon}^{\mathbf{k}}} \mathbf{u}_{\varepsilon} \cdot \mathbf{n}_{\varepsilon}
$$

where $\mathbf{n}_{\varepsilon}$ is the unit normal to $\partial \Omega_{\varepsilon}$, pointing into the gaseous bubbles.

The equation of motion for the incompressible viscoelastic structure is given by

$$
\left\{\begin{aligned}
\rho_{s} \frac{\partial^{2} \mathbf{u}_{\varepsilon}}{\partial t^{2}} & =\operatorname{div} \sigma_{\varepsilon}+\mathbf{f} \\
\operatorname{div} \mathbf{u}_{\varepsilon} & =0
\end{aligned}\right.
$$

where $\rho_{s}>0$ denotes the density of the alveolar wall, $\mathbf{f}$ is a volumic excitation force, and $\sigma_{\varepsilon}$ is the stress tensor, which is related to $\nabla \mathbf{u}_{\varepsilon}$ by the constitutive law of the inhomogeneous wall material. We wish to study specifically the possible effects of the heterogeneity of the alveolar wall. In order to describe linear viscoelastic behavior, we recover the stress response from the strain history input as follows:

$$
\sigma_{\varepsilon}(\mathbf{x}, t)=-p_{\varepsilon}(\mathbf{x}, t) \operatorname{Id}+\int_{-\infty}^{t} E_{\varepsilon}(\mathbf{x}, t-\tau) \frac{\partial}{\partial \tau} e\left(\mathbf{u}_{\varepsilon}(\mathbf{x}, \tau)\right) \mathrm{d} \tau
$$


where

$\sigma_{\varepsilon}$ is the stress tensor;

$p_{\varepsilon}$ is a Lagrange multiplier associated with the constraint $\operatorname{div} \mathbf{u}_{\varepsilon}=0$;

$E_{\varepsilon}$ is the relaxation function, a fourth-order time-dependent tensor;

$e\left(\mathbf{u}_{\varepsilon}\right)=1 / 2\left(\nabla \mathbf{u}_{\varepsilon}+{ }^{T} \nabla \mathbf{u}_{\varepsilon}\right)$ is the strain tensor of the material;

Id is the fourth order identity tensor.

Note that $p_{\varepsilon}$ is homogeneous to a pressure. At the boundary of the gaseous bubbles we have balance of the forces, that is

$$
\mathbf{n}_{\varepsilon} \cdot \sigma_{\varepsilon}=\left(p_{a}-p_{\varepsilon}^{\mathbf{k}}\right) \mathbf{n}_{\varepsilon} \approx-\frac{\gamma p_{a}}{\left|\mathcal{B}_{\varepsilon}^{\mathbf{k}}\right|}\left(\int_{\partial \mathcal{B}_{\varepsilon}^{\mathbf{k}}} \mathbf{u}_{\varepsilon} \cdot \mathbf{n}_{\varepsilon}\right) \mathbf{n}_{\varepsilon} \quad \text { on each } \partial \mathcal{B}_{\varepsilon}^{\mathbf{k}} .
$$

For simplicity, we suppose that the material is initially at rest at time $t=0$ and we impose homogeneous Dirichlet boundary conditions on the exterior boundary $\partial \Omega$. We collect the equations for the structure (1.2) and (1.3) as well as the boundary constraint describing the bubble response (1.4) to recover the following microscale description of the model:

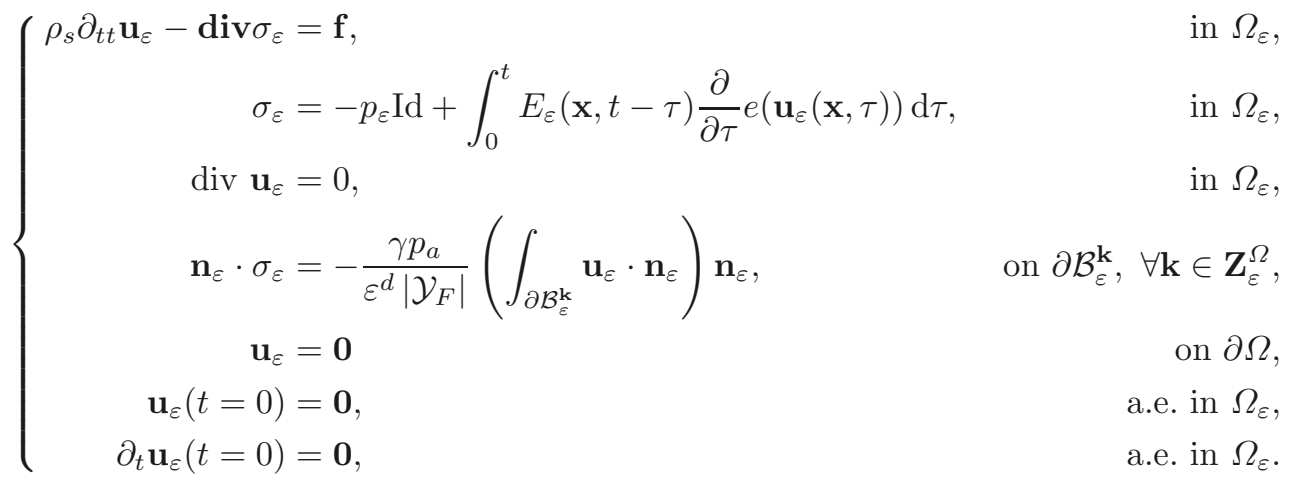

\subsubsection{Heterogeneities}

Following [14], the relaxation function writes

$$
E_{\varepsilon}(\mathbf{x}, t)=E_{\infty}(\mathbf{x} / \varepsilon)+G(\mathbf{x} / \varepsilon, t)=E_{\infty}(\mathbf{y})+G(\mathbf{y}, t),
$$

where $E_{\infty}$ represents the elastic response of the material at equilibrium, while $G$ denotes the memory properties of the material. We suppose that $E_{\infty}(\mathbf{y})$ and $G(\mathbf{y}, t)$ belong respectively to $\mathrm{L}^{\infty}(\mathcal{Y}, S y m)$ and $\mathrm{L}^{1}\left(\mathbb{R}^{+} ; \mathrm{L}^{\infty}(\mathcal{Y}\right.$, Sym $\left.)\right)$ where Sym is the set of symmetric fourth-order tensors. Furthermore, we suppose that $E_{\infty}$ is uniformly definite positive, meaning that there exists $\alpha>0$ such that for all $\mathbf{y} \in \mathcal{Y}$ and any symmetric matrix $\xi$,

$$
\sum_{1 \leq i, j \leq d} E_{\infty, i j k l}(\mathbf{y}) \xi_{i j} \xi_{k l} \geq \alpha|\xi|^{2}
$$

In addition, the time-dependent tensor $G$ is positive and satisfies the weak dissipativity condition [14]:

$$
\sum_{1 \leq i, j \leq d} \int_{-\infty}^{T} \int_{-\infty}^{t} G_{i j k l}(t-s) \xi_{i j}(s) \xi_{k l}(t) \geq 0,
$$

for all smooth test functions with compact support $\xi(t)$ in Sym and $T>0$. Modeling the structure as a network of elastic fibers coated with a viscous gel representing the ground substance, we expect the elastic coefficients $E_{\infty}$ to dominate in the fibers while the viscous components $G$ dominate in the gel. Other models are also possible, such as modeling the gel as a purely Newtonian fluid and the fibers as a purely elastic medium (see v.g. [12]). 


\subsection{The mathematical homogenization method}

In this section, we outline the mathematical homogenization procedure to derive equations for the macroscopic motion of the medium. Clearly, it is not possible to solve (1.5) on a realistic geometry for more than a small number of alveoli, and certainly not for the millions that are contained in the parenchyma. To derive effective equations, the two-scale method of homogenization is a widely used tool that has previously been heuristically applied to different models of the lung parenchyma by Owen and Lewis [28] as well as Siklosi et al. [35] for example. The approach consists in modeling the parenchyma as an array of periodically repeating cells, representing individual alveoli, and obtain equations governing the behavior of spatially averaged relevant quantities such as deformation and pressure by separating the variations at the micro-scale and macro-scale. It is also possible to deal with random inhomogeneities in the micro-structure [6], but the evaluation of the effective parameters is more complicated.

\subsubsection{Weak formulation, existence and uniqueness}

First, we study the existence and uniqueness of weak solutions to (1.5). For such viscoelastic problems this has been proved in a slightly different context [11]. Define the Sobolev spaces $\mathbf{V}_{\varepsilon}=\mathbf{H}_{0}^{1}\left(\Omega_{\varepsilon}\right), \mathbf{X}_{\varepsilon}=\mathbf{L}^{2}\left(\Omega_{\varepsilon}\right)$, $\mathrm{M}_{\varepsilon}=\mathrm{L}^{2}\left(\Omega_{\varepsilon}\right)$, and $\mathbf{V}=\mathbf{H}_{0}^{1}(\Omega), \mathbf{X}=\mathbf{L}^{2}(\Omega), \mathrm{M}=\mathrm{L}^{2}(\Omega)$. Let $T>0$, the variational formulation associated with (1.5) is as follows.

Find $\mathbf{u}_{\varepsilon}$ in $\mathrm{L}^{\infty}\left(0, T ; \mathbf{V}_{\varepsilon}\right), \partial_{t} \mathbf{u}_{\varepsilon} \in \mathrm{L}^{\infty}\left(0, T ; \mathbf{X}_{\varepsilon}\right), p_{\varepsilon} \in \mathrm{H}^{-1}\left(0, T ; \mathrm{M}_{\varepsilon}\right)$ such that for all $\mathbf{v}_{\varepsilon} \in \mathbf{V}_{\varepsilon}$,

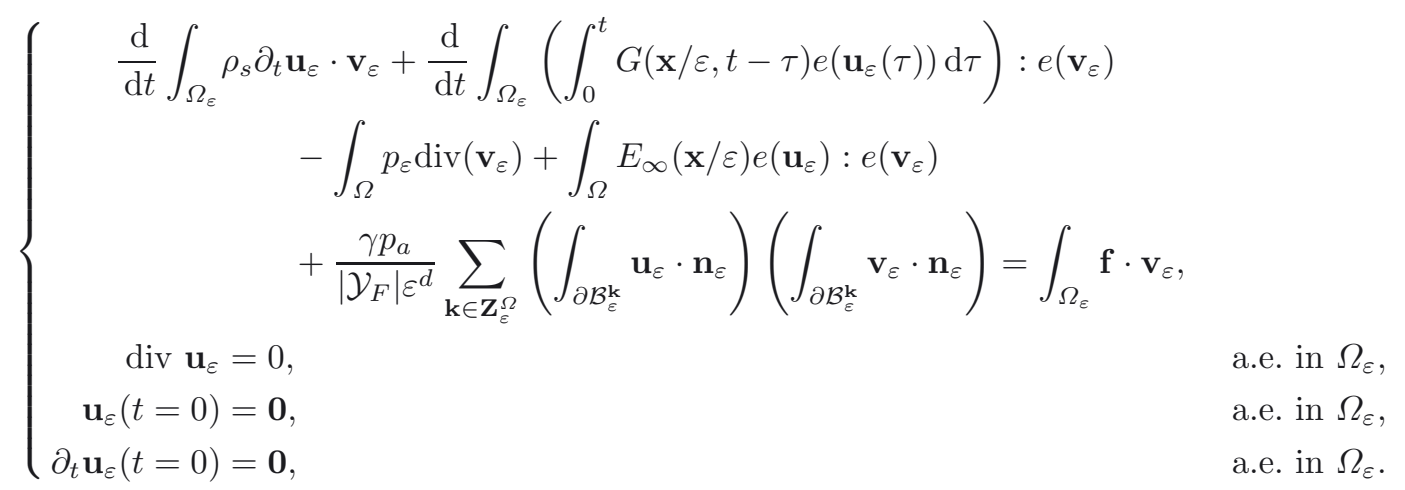

This formulation has to be understood in $\mathrm{H}^{-1}(0, T)$. Thanks to the positivity condition (1.7) we have the following result, for which an idea of the proof is given in Appendix A:

Proposition 1.1. Assume that $\mathbf{f} \in \mathrm{L}^{2}(0, T ; \mathbf{X})$.

Then, there exists a unique $\mathbf{u}_{\varepsilon} \in \mathrm{L}^{\infty}\left(0, T ; \mathbf{V}_{\varepsilon}\right) \cap \mathrm{W}^{1, \infty}\left(0, T ; \mathbf{X}_{\varepsilon}\right)$ and $p_{\varepsilon} \in \mathrm{H}^{-1}\left(0, T ; \mathrm{M}_{\varepsilon}\right)$ which satisfies (1.9). Moreover, there exists a constant $C$ which does not depend on $\varepsilon$ such that:

$$
\left\|\partial_{t} \mathbf{u}_{\varepsilon}\right\|_{L^{\infty}\left(0, T ; \mathbf{X}_{\varepsilon}\right)}+\left\|\mathbf{u}_{\varepsilon}\right\|_{L^{\infty}\left(0, T ; \mathbf{V}_{\varepsilon}\right)}+\left\|p_{\varepsilon}\right\|_{H^{-1}\left(0, T ; \mathrm{M}_{\varepsilon}\right)} \leq C\|\mathbf{f}\|_{L^{2}\left(0, T ; \mathbf{X}_{\varepsilon}\right)}
$$

If the excitation force $\mathbf{f}$ satisfies a stronger regularity assumption, one obtains a better regularity for the solution:

Proposition 1.2. Suppose that $\mathbf{f} \in \mathrm{H}^{1}(0, T ; \mathbf{X})$. Then, there exists $\mathbf{u}_{\varepsilon}$ in $\mathrm{W}^{1, \infty}\left(0, T ; \mathbf{V}_{\varepsilon}\right) \cap \mathrm{W}^{2, \infty}\left(0, T ; \mathbf{X}_{\varepsilon}\right)$ and $p_{\varepsilon} \in \mathrm{L}^{\infty}\left(0, T ; \mathrm{M}_{\varepsilon}\right)$ which satisfies (1.9). Moreover, there exists a constant $C$ which does not depend on $\varepsilon$ such that:

$$
\left\|\mathbf{u}_{\varepsilon}\right\|_{\mathrm{W}^{2, \infty}\left(0, T ; \mathbf{X}_{\varepsilon}\right)}+\left\|\mathbf{u}_{\varepsilon}\right\|_{\mathrm{W}^{1, \infty}\left(0, T ; \mathbf{V}_{\varepsilon}\right)}+\left\|p_{\varepsilon}\right\|_{\mathrm{L}^{\infty}\left(0, T ; \mathrm{M}_{\varepsilon}\right)} \leq C\|\mathbf{f}\|_{\mathrm{H}^{1}\left(0, T ; \mathbf{X}_{\varepsilon}\right)} .
$$

The proof consists simply in differentiating the weak formulation in time and using the result of Proposition 1.1. The $\mathrm{L}^{\infty}$-bound on the pressure is obtained thanks to an inf-sup condition uniform in $\varepsilon$, see Proposition 3.1 in [2]. 


\subsubsection{Asymptotic behavior}

Hereafter, we suppose that $\mathbf{f} \in \mathrm{H}^{1}(0, T ; \mathbf{X})$ for simplicity of exposition. We now study the properties of the solution of the system as $\varepsilon$ converges to zero. This procedure allows us to average out the microscale oscillations to understand the macroscale properties of the solution. We use here the notion of two-scale convergence introduced by Nguetseng [27] and Allaire [1]. We denote by $\widetilde{r}$ the extension by zero in $\Omega \backslash \Omega_{\varepsilon}$ and we adopt the following convention: the index \# denotes spaces of $\mathcal{Y}$-periodic functions on $\mathcal{Y}$ or $\mathcal{Y}_{S}$ with null average, in particular we set

$$
\mathbf{H}_{\#}^{1}\left(\mathcal{Y}_{S}\right)=\left\{\mathbf{v}^{1} \in \mathrm{H}^{1}\left(\mathcal{Y}_{S} ; \mathbb{R}^{d}\right), \quad \mathbf{v}^{1} \text { is } \mathcal{Y} \text {-periodic and } \int_{\mathcal{Y}_{S}} \mathbf{v}^{1}=\mathbf{0}\right\} .
$$

We extend naturally the definition of two-scale convergence to the time-dependent setting, as in [12] .

Definition 1.3. A sequence $\left(u_{\varepsilon}(t, \mathbf{x})\right)_{\varepsilon>0}$ in $\mathrm{L}^{2}((0, T) \times \Omega)$ two-scale converges to $u(t, \mathbf{x}, \mathbf{y})$ in $\mathrm{L}^{2}((0, T) \times \Omega \times \mathcal{Y})$ if for any $v(t, \mathbf{x}, \mathbf{y})$ in $\mathrm{L}^{2}\left((0, T) \times \Omega, C_{\#}(\mathcal{Y})\right)$, one has:

$$
\lim _{\varepsilon \rightarrow 0} \int_{0}^{T} \int_{\Omega} u_{\varepsilon}(t, \mathbf{x}) v(t, \mathbf{x}, \mathbf{x} / \varepsilon) \mathrm{d} x \mathrm{~d} t=\int_{0}^{T} \int_{\Omega} \int_{\mathcal{Y}} u(t, \mathbf{x}, \mathbf{y}) v(t, \mathbf{x}, \mathbf{y}) \mathrm{d} y \mathrm{~d} x \mathrm{~d} t .
$$

Thanks to the compactness properties of the two-scale convergence (see v.g. [1]), it is well-known that the a priori bounds (1.10) imply the existence of three functions $\mathbf{u}$ in $\mathrm{H}^{1}(0, T ; \mathbf{V})$ with $\partial_{t} \mathbf{u}$ in $\mathrm{H}^{1}(0, T ; \mathbf{X})$, $\mathbf{u}^{1}$ in $\mathrm{H}^{1}\left(0, T ; \mathrm{L}^{2}\left(\Omega, \mathbf{H}_{\#}^{1}\left(\mathcal{Y}_{S}\right)\right)\right)$ and $p \in \mathrm{L}^{2}\left((0, T) \times \Omega \times \mathcal{Y}_{S}\right)$ such that, up to a subsequence:

$$
\left\{\begin{aligned}
\widetilde{\mathbf{u}_{\varepsilon}} & \rightarrow \mathbf{u}(\mathbf{x}) \chi_{S}(\mathbf{y}) & & \text { in the two-scale sense } \\
\widetilde{\nabla \mathbf{u}_{\varepsilon}} & \rightarrow\left(\nabla_{x} \mathbf{u}(\mathbf{x})+\nabla_{y} \mathbf{u}^{1}(\mathbf{x}, \mathbf{y})\right) \chi_{S}(\mathbf{y}) & & \text { in the two-scale sense } \\
\widetilde{p_{\varepsilon}} & \rightarrow p(\mathbf{x}, \mathbf{y}) \chi_{S}(\mathbf{y}) & & \text { in the two-scale sense }
\end{aligned}\right.
$$

where $\chi_{S}$ is the characteristic function of $\mathcal{Y}_{S}$.

Remark 1.4. Note that the convergence results (1.11) are a rigorous expression of the heuristic argument that the properties of the material can be expanded as an asymptotic expansion in powers of $\varepsilon$ :

$$
\mathbf{u}_{\varepsilon}(\mathbf{x}, t) \approx \mathbf{u}(\mathbf{x}, t)+\varepsilon \mathbf{u}^{1}(\mathbf{x}, \mathbf{x} / \varepsilon, t)+\varepsilon^{2} \mathbf{u}^{2}(\mathbf{x}, \mathbf{x} / \varepsilon, t)+\ldots
$$

We are now in a position to pass to the limit in the variational formulation (1.9). The idea is to use oscillating test functions of the form

$$
\mathbf{v}_{\varepsilon}=\mathbf{v}(\mathbf{x})+\varepsilon \mathbf{v}^{1}(\mathbf{x}, \mathbf{x} / \varepsilon) .
$$

Thanks to the two-scale convergences (1.11) we can pass to the limit in most terms of the weak formulation (1.9). We refer to [2] for details on the specific treatment of the term containing a non-local product of integrals in (1.9). In the end, we obtain that $\mathbf{u}, \mathbf{u}^{1}$ and $p$ are solutions of the variational formulation, for all $\mathbf{v} \in \mathbf{V}$ and $\mathbf{v}^{1} \in \mathrm{L}^{2}\left(\Omega, \mathrm{H}_{\#}^{1}\left(\mathcal{Y}_{S}\right)\right)$ :

$$
\left\{\begin{array}{l}
\int_{\Omega} \int_{\mathcal{Y}_{S}} \rho_{s} \partial_{t t} \mathbf{u} \cdot \mathbf{v} \\
\quad+\int_{\Omega} \int_{\mathcal{Y}_{S}}\left(\int_{0}^{t} G(\mathbf{y}, t-\tau)\left(e_{\mathbf{x}}\left(\partial_{t} \mathbf{u}(\tau)\right)+e_{\mathbf{y}}\left(\partial_{t} \mathbf{u}^{1}(\tau)\right)\right) \mathrm{d} \tau\right):\left(e_{\mathbf{x}}(\mathbf{v})+e_{\mathbf{y}}\left(\mathbf{v}^{1}\right)\right) \\
\quad-\int_{\Omega} \int_{\mathcal{Y}_{S}} p\left(\operatorname{div}_{\mathbf{x}}(\mathbf{v})+\operatorname{div}_{\mathbf{y}}\left(\mathbf{v}^{1}\right)\right) \\
\quad+\int_{\Omega} \int_{\mathcal{Y}_{S}} E_{\infty}(\mathbf{y})\left(e_{\mathbf{x}}(\mathbf{u})+e_{\mathbf{y}}\left(\mathbf{u}^{1}\right)\right):\left(e_{\mathbf{x}}(\mathbf{v})+e_{\mathbf{y}}\left(\mathbf{v}^{1}\right)\right) \\
\quad+\frac{|\mathcal{Y}| \gamma p_{a}}{\left|\mathcal{Y}_{F}\right|} \int_{\Omega} \operatorname{div}_{\mathbf{x}} \mathbf{u}\left(\left|\mathcal{Y}_{F}\right| \operatorname{div}_{\mathbf{x}} \mathbf{v}-\int_{\Gamma} \mathbf{v}^{1} \cdot \mathbf{n}\right)=\int_{\Omega} \int_{\mathcal{Y}_{S}} \mathbf{f} \cdot \mathbf{v}, \quad \\
\quad \operatorname{div}_{\mathbf{x}} \mathbf{u}+\operatorname{div}_{\mathbf{y}} \mathbf{u}^{1}=0, \quad \mathbf{u}(t=0)=\mathbf{0}, \quad \partial_{t} \mathbf{u}(t=0)=\mathbf{0}, \quad \text { a.e. in } \Omega .
\end{array}\right.
$$


Proposition 1.5. The system (1.12) has a unique solution with $\mathbf{u} \in \mathrm{W}^{1, \infty}(0, T ; \mathbf{V}) \cap \mathrm{W}^{2, \infty}(0, T ; \mathbf{X}), \mathbf{u}^{1} \in$ $\mathrm{W}^{1, \infty}\left(0, T ; \mathrm{L}^{2}\left(\Omega, \mathbf{H}_{\#}^{1}\left(\mathcal{Y}_{S}\right)\right)\right.$ and $p \in \mathrm{L}^{\infty}\left((0, T) \times \Omega \times \mathcal{Y}_{S}\right)$. Also, the whole sequence $\left(\widetilde{\mathbf{u}_{\varepsilon}}, \widetilde{p_{\varepsilon}}\right)_{\varepsilon>0}$ two-scale converges to $\mathbf{u}, \mathbf{u}^{1}$ and $p$ in the sense of (1.11).

Proof. The proof of this result follows that of Proposition 1.1, given that $\left\|e_{\mathbf{x}}(\mathbf{u})+e_{\mathbf{y}}\left(\mathbf{u}^{1}\right)\right\|_{\mathrm{L}^{2}\left(\Omega \times \mathcal{Y}_{S}\right)}$ is a norm for the Hilbert space $\mathbf{V} \times \mathrm{L}^{2}\left(\Omega ; \mathbf{H}_{\#}^{1}\left(\mathcal{Y}_{S}\right)\right)$ (Lem. 2.5 in [2]). Existence and uniqueness of the limit pressure $p$ can be proved using a two-scale inf-sup condition (Lem. 3.6 in [2]).

\subsection{The microcell problem}

The two-scale homogenized system is too complicated to be used directly. We are going to eliminate the micro-scale variables $\mathbf{u}^{1}$ and $p$ to obtain the effective macro-scale equations. The system (1.12) is linear, and admits a unique solution. Hence, because of the superposition principle, we seek to express formally $\mathbf{u}^{1}(\mathbf{x}, \mathbf{y}, t)$ and $p(\mathbf{x}, \mathbf{y}, t)$ as expressions parameterized by the macro-scale displacement history $(\mathbf{u}(\mathbf{x}, \tau))_{\tau \leq t}$ :

$$
\left\{\begin{aligned}
\mathbf{u}^{1}(\mathbf{x}, \mathbf{y}, t) & =\sum_{1 \leq i, j \leq d} \int_{0}^{t} \chi^{i j}(\mathbf{y}, t-\tau) e_{\mathbf{x}}(\mathbf{u})_{i j}(\mathbf{x}, \tau) \mathrm{d} \tau, \\
p(\mathbf{x}, \mathbf{y}, t) & =\sum_{1 \leq i, j \leq d} \int_{0}^{t} \eta^{i j}(\mathbf{y}, t-\tau) e_{\mathbf{x}}(\mathbf{u})_{i j}(\mathbf{x}, \tau) \mathrm{d} \tau,
\end{aligned}\right.
$$

where $\chi^{i j}(\mathbf{y}, t)$ and $\eta^{i j}(\mathbf{y}, t)$ are correctors to be determined as solutions of cell problems set on $\mathcal{Y}_{S}$. Note that $\chi^{i j}$ and $\eta^{i j}$ are in general distributions in the time domain, and obtaining their precise expression from the timedependent two-scale problem (1.12) is possible but requires cumbersome calculations (see $[11,12]$ for a similar derivation). To better understand the frequency-domain behavior of the effective medium, and to simplify the analysis, we use the Fourier transform in the time variable to describe the local problems. For a given integrable or square-integrable function $\psi$, we denote its (complex-valued) Fourier transform $\widehat{\psi}$ defined for any $\omega \in \mathbb{R}$ as

$$
\widehat{\psi}(\omega)=\frac{1}{\sqrt{2 \pi}} \int_{-\infty}^{\infty} \psi(t) \mathrm{e}^{-i \omega t} \mathrm{~d} t .
$$

If $\psi$ is defined on $(0, T)$, we extend it by zero elsewhere. Taking the Fourier transform and setting $\mathbf{v}=0$ in (1.12), we obtain that the functions $\widehat{\mathbf{u}^{1}}$ and $\widehat{p}$ are solutions of the following variational problem parameterized by $e_{\mathbf{x}}(\widehat{\mathbf{u}})$ for any frequency $\omega$ : for all $\mathbf{v}^{1} \in \mathbf{H}_{\#}^{1}\left(\mathcal{Y}_{S}\right)$,

$$
\left\{\begin{array}{r}
\int_{\mathcal{Y}_{S}}\left(E_{\infty}+i \omega \widehat{G}(\omega)\right)\left(e_{\mathbf{x}}(\widehat{\mathbf{u}})+e_{\mathbf{y}}\left(\widehat{\mathbf{u}^{1}}\right)\right): e_{\mathbf{y}}\left(\mathbf{v}^{1}\right)-\int_{\mathcal{Y}_{S}} \widehat{p} \operatorname{div}_{\mathbf{y}}\left(\mathbf{v}^{1}\right)-\frac{|\mathcal{Y}| \gamma p_{a}}{\left|\mathcal{Y}_{F}\right|} \operatorname{div}_{\mathbf{x}} \widehat{\mathbf{u}} \int_{\Gamma} \mathbf{v}^{1} \cdot \mathbf{n}=\mathbf{0} \\
\operatorname{div}_{\mathbf{x}} \widehat{\mathbf{u}}+\operatorname{div}_{\mathbf{y}} \widehat{\mathbf{u}^{1}}=0 .
\end{array}\right.
$$

Note that, by Stokes formula, $\int_{\Gamma} \mathbf{v}^{1} \cdot \mathbf{n}=\int_{\mathcal{Y}_{S}} \operatorname{div}_{\mathbf{y}} \mathbf{v}^{1}$. Substituting the two decompositions (1.13), we have, collecting the terms in $e_{\mathbf{x}}(\widehat{\mathbf{u}})$,

$$
\left\{\begin{array}{r}
\left(\int_{\mathcal{Y}_{S}}\left(E_{\infty}+i \omega \widehat{G}(\omega)\right) e_{\mathbf{y}}\left(\mathbf{p}^{i j}+\widehat{\chi^{i j}}(\omega)\right): e_{\mathbf{y}}\left(\mathbf{v}^{1}\right)-\int_{\mathcal{Y}_{S}}\left(\frac{|\mathcal{Y}| \gamma p_{a}}{\left|\mathcal{Y}_{F}\right|} \delta_{i j}+\widehat{\eta^{i j}}(\omega)\right) \operatorname{div}_{\mathbf{y}}\left(\mathbf{v}^{1}\right)\right) e_{\mathbf{x}}(\widehat{\mathbf{u}})_{i j}=\mathbf{0}, \\
\left(\delta_{i j}+\operatorname{div}_{\mathbf{y}} \widehat{\chi^{i j}}(\omega)\right) e_{\mathbf{x}}(\widehat{\mathbf{u}})_{i j}=0
\end{array}\right.
$$


where we have introduced the family of polynomials $\mathbf{p}^{i j}, 1 \leq i, j \leq d$ defined as:

$$
\mathbf{p}^{i j}(\mathbf{y})=\frac{1}{2}\left(y_{i} \mathbf{e}^{j}+y_{j} \mathbf{e}^{i}\right),
$$

which is chosen so that the family $e_{\mathbf{y}}\left(\mathbf{p}^{i j}\right)=\frac{1}{2}\left(\mathbf{e}^{i} \otimes \mathbf{e}^{j}+\mathbf{e}^{j} \otimes \mathbf{e}^{i}\right)$ constitutes a basis of the set of symmetric tensors.

Hence, it is natural to seek $\widehat{\chi^{i j}}(\omega, \mathbf{y})$ and $\widehat{\eta^{i j}}(\omega, \mathbf{y})$ as solutions of mixed cell problems parameterized by $\omega$ :

$$
\left\{\begin{array}{rlrl}
-\operatorname{div}_{\mathbf{y}} \sigma_{y}^{\omega}\left(\widehat{\chi^{i j}}(\omega), \widehat{\eta^{i j}}(\omega)\right) & =\operatorname{div}_{\mathbf{y}} \sigma_{y}^{\omega}\left(\mathbf{p}^{i j}, \frac{|\mathcal{Y}| \gamma p_{a}}{\left|\mathcal{Y}_{F}\right|} \delta_{i j}\right), & & \text { in } \mathcal{Y}_{S}, \\
\operatorname{div}_{\mathbf{y}} \widehat{\chi^{i j}}(\omega) & =-\delta_{i j}, & & \text { in } \mathcal{Y}_{S}, \\
\sigma_{y}^{\omega}\left(\widehat{\chi^{i j}}(\omega), \widehat{\eta^{i j}}(\omega)\right) \mathbf{n} & =-\sigma_{y}^{\omega}\left(\mathbf{p}^{i j}, \frac{|\mathcal{Y}| \gamma p_{a}}{\left|\mathcal{Y}_{F}\right|} \delta_{i j}\right) \mathbf{n}, & & \text { on } \Gamma, \\
\widehat{\eta^{i j}}(\omega), \widehat{\chi^{i j}}(\omega) \mathcal{Y} \text {-periodic, } & &
\end{array}\right.
$$

where the local stress tensor $\sigma_{y}^{\omega}$ is defined for any $\omega \in \mathbb{R}$ as

$$
\sigma_{y}^{\omega}(\chi, \eta)=-\eta \operatorname{Id}+\left(E_{\infty}+i \omega \widehat{G}(\omega)\right) e_{\mathbf{y}}(\chi)
$$

To ensure that this problem is well-posed, we make hereafter the assumption that for any frequency $\omega$, there exists a constant $\alpha(\omega)>0$ such that

$$
\left|\left(E_{\infty}(\mathbf{y})+i \omega \widehat{G}(\mathbf{y}, \omega)\right) \xi: \xi\right| \geq \alpha(\omega)|\xi|^{2} \quad \text { for any } \mathbf{y} \in \mathcal{Y}_{S} \text { and } \xi \in \text { Sym }
$$

Existence and uniqueness of the correctors $\widehat{\chi^{i j}}(\omega) \in \mathbf{H}_{\#}^{1}\left(\mathcal{Y}_{S}\right)$ and $\widehat{\eta^{i j}}(\omega) \in \mathrm{L}_{\#}^{2}\left(\mathcal{Y}_{S}\right)$ as solutions of (1.14) is then a consequence of the Lax-Milgram theorem.

Remark 1.6. The quantity $E_{\infty}(\mathbf{y})+i \omega \widehat{G}(\mathbf{y}, \omega)$ is called the complex relaxation modulus, and is the frequencydomain equivalent of the relaxation function in the description of the viscoelastic material. Condition (1.15) is satisfied in particular if the real or imaginary part of the complex relaxation modulus is positive definite. For $\omega=0$ we recover the condition (1.7) satisfied by any solid material. For $\omega>0$, we know in general that the dissipation condition (1.8) implies that the imaginary part $\omega \operatorname{Re}(\widehat{G}(\mathbf{y}, \omega))$ of the complex relaxation modulus is always a positive tensor, and is positive definite for any truly dissipative viscoelastic material (see v.g. [14]). Note that a lower bound $\alpha(\omega) \geq \alpha_{-}>0$ can be obtained by a continuity argument if (1.15) holds and the limit $\lim _{\omega \rightarrow \infty} \omega \widehat{G}(\mathbf{y}, \omega)$ exists. However, this is not true in the general case (see [10], Chap. 7).

Remark 1.7. It is well-known that the solutions of (1.14) depend continuously on the parameters. Since $G(\mathbf{y}, t)$ belongs to $\mathrm{L}^{1}(0, T)$, its Fourier transform $\widehat{G}(\mathbf{y}, \omega)$ is a continuous function of $\omega$, hence the functions $\omega \mapsto \widehat{\chi^{i j}}(\omega)$ and $\omega \mapsto \widehat{\eta^{i j}}(\omega)$ are also continuous.

\subsection{Effective equation and effective relaxation modulus}

We have seen that for a given $\widehat{\mathbf{u}}$, the local variables $\widehat{\mathbf{u}^{1}}$ and $\widehat{p}$ are determined by the formulae

$$
\widehat{\mathbf{u}^{1}}(\mathbf{x}, \mathbf{y}, \omega)=\sum_{1 \leq i, j \leq d} e_{\mathbf{x}}(\widehat{\mathbf{u}})_{i j}(\mathbf{x}, \omega) \widehat{\chi^{i j}}(\mathbf{y}, \omega), \quad \widehat{p}(\mathbf{x}, \mathbf{y}, \omega)=\sum_{1 \leq i, j \leq d} e_{\mathbf{x}}(\widehat{\mathbf{u}})_{i j}(\mathbf{x}, \omega) \widehat{\eta^{i j}}(\mathbf{y}, \omega) .
$$


Applying the Fourier transform to the system (1.12) and taking $\mathbf{v}^{1}=0$, we obtain

$$
\left\{\begin{aligned}
\int_{\Omega}-\rho_{s}\left|\mathcal{Y}_{S}\right| \omega^{2} \widehat{\mathbf{u}} \cdot \mathbf{v}+ & \int_{\Omega} e_{\mathbf{x}}(\widehat{\mathbf{u}})_{i j}(\mathbf{x})\left(\int_{\mathcal{Y}_{S}}\left(E_{\infty}+i \omega \widehat{G}(\omega)\right) e_{\mathbf{y}}\left(\mathbf{p}^{i j}+\widehat{\chi}^{i j}\right)\right): e_{\mathbf{x}}(\mathbf{v}) \\
& -\int_{\Omega} e_{\mathbf{x}}(\widehat{\mathbf{u}})_{i j}(\mathbf{x})\left(\int_{\mathcal{Y}_{S}} \widehat{\eta^{i j}}\right) \operatorname{div}_{\mathbf{x}}(\mathbf{v})+|\mathcal{Y}| \gamma p_{a} \int_{\Omega} \operatorname{div}_{\mathbf{x}} \widehat{\mathbf{u}} \operatorname{div}_{\mathbf{x}} \mathbf{v}=\int_{\Omega}\left|\mathcal{Y}_{S}\right| \widehat{\mathbf{f}} \cdot \mathbf{v}
\end{aligned}\right.
$$

Hence the effective equation in the frequency domain for the displacement of the structure $\widehat{\mathbf{u}}$ takes the form

$$
-\omega^{2}\left|\mathcal{Y}_{S}\right| \rho_{S} \widehat{\mathbf{u}}-\operatorname{div}\left(\widehat{\mathcal{G}}^{h o m} e(\widehat{\mathbf{u}})\right)=\left|\mathcal{Y}_{S}\right| \widehat{\mathbf{f}}
$$

where the homogenized complex relaxation modulus of the material $\widehat{\mathcal{G}}^{\text {hom }}$ is obtained by averaging the correctors in $\mathcal{Y}_{S}$ :

$$
\widehat{\mathcal{G}}_{i j k l}^{h o m}(\omega)=\left|\mathcal{Y}_{S}\right|\left(E_{\infty}+i \omega \widehat{G}(\omega)+\frac{|\mathcal{Y}| \gamma p_{a}}{\left|\mathcal{Y}_{S}\right|} \mathrm{Id}\right)_{i j k l}+\left(\int_{\mathcal{Y}_{S}} \sigma_{y}^{\omega}\left(\widehat{\chi}^{i j}(\omega), \eta^{i j}(\omega)\right)\right)_{k l}
$$

It is easily shown (see $v \cdot g$. [1]) that $\widehat{\mathcal{G}}^{\text {hom }}$ satisfies the usual symmetry conditions, for any value of $\omega$ :

$$
\widehat{\mathcal{G}}_{i j k l}^{h o m}=\widehat{\mathcal{G}}_{k l i j}^{h o m}=\widehat{\mathcal{G}}_{i j l k}^{h o m} .
$$

In addition, the data and coefficients in (1.14) are real when $\omega=0$, hence $\widehat{\mathcal{G}}^{\text {hom }}(0)$ is positive definite.

\subsubsection{Decomposition of the relaxation modulus}

To come back to the time-domain, it is necessary to further decompose the complex relaxation modulus $\widehat{\mathcal{G}}^{\text {hom }}$. By analogy with (1.6), we introduce the homogenized equilibrium modulus $E_{\infty}^{\text {hom }}=\widehat{\mathcal{G}}^{\text {hom }}(0)$ and write

$$
\widehat{\mathcal{G}}^{h o m}(\omega)=E_{\infty}^{h o m}+i \omega \widehat{G}^{h o m}(\omega)
$$

To compute the memory function $G^{h o m}$, let us also decompose $\widehat{\chi^{i j}}$ and $\widehat{\eta^{i j}}$ as

$$
\widehat{\chi^{i j}}(\omega)=\widehat{\chi^{i j}}(0)+i \omega \widehat{\phi^{i j}}(\omega), \quad \widehat{\eta^{i j}}(\omega)=\widehat{\eta^{i j}}(0)+i \omega \widehat{\psi^{i j}}(\omega) .
$$

By substraction in (1.14), the functions $\widehat{\phi^{i j}}(\omega)$ and $\widehat{\psi^{i j}}(\omega)$ are solutions of the mixed problem:

$$
\left\{\begin{array}{rlrl}
-\operatorname{div}_{\mathbf{y}} \sigma_{y}^{\omega}\left(\widehat{\phi^{i j}}(\omega), \widehat{\psi^{i j}}(\omega)\right) & =\operatorname{div}_{\mathbf{y}}\left(\widehat{G}(\omega)\left(\mathbf{p}^{i j}+\widehat{\chi^{i j}}(0)\right)\right) & & \text { in } \mathcal{Y}_{S}, \\
\operatorname{div}_{\mathbf{y}} \widehat{\phi^{i j}}(\omega) & =0 & & \text { in } \mathcal{Y}_{S}, \\
\sigma_{y}^{\omega}\left(\widehat{\phi^{i j}}(\omega), \widehat{\psi^{i j}}(\omega)\right) \mathbf{n} & =-\left(\widehat{G}(\omega) e_{\mathbf{y}}\left(\mathbf{p}^{i j}+\widehat{\chi^{i j}}(0)\right)\right) \mathbf{n} & & \text { on } \Gamma, \\
\widehat{\psi^{i j}}, \widehat{\phi^{i j}} \mathcal{Y} \text {-periodic. } & &
\end{array}\right.
$$

Now the effective memory function $G^{\text {hom }}$ is defined by its Fourier transform as:

$$
\widehat{G}_{i j k l}^{h o m}(\omega)=\int_{\mathcal{Y}_{S}} \widehat{G}_{i j k l}(\omega)+\left(\int_{\mathcal{Y}_{S}} \widehat{G}(\omega) e_{\mathbf{y}}\left(\widehat{\chi}^{i j}(0)\right)+\sigma_{y}^{\omega}\left(\widehat{\phi}^{i j}(\omega), \psi^{i j}(\omega)\right)\right)_{k l} .
$$




\subsection{Effective equations in the time domain}

Let us now conclude this presentation of the homogenization process by describing the effective behavior of the viscoelastic porous domain as a function of time. The macroscopic displacement is described by the variable $\mathbf{u}(\mathbf{x}, t)$, and combining (1.16) and (1.18), we obtain the homogenized system

$$
\left\{\begin{aligned}
\left|\mathcal{Y}_{S}\right| \rho_{S} \partial_{t t} \mathbf{u}(t) & -\operatorname{div}\left(\sigma^{\text {hom }}(t)\right)=\left|\mathcal{Y}_{S}\right| \mathbf{f}(t), & & \text { in } \Omega, \\
\sigma^{h o m}(t) & =E_{\infty}^{h o m} e(\mathbf{u}(t))+\int_{0}^{t} G^{h o m}(t-\tau) e\left(\partial_{t} \mathbf{u}(\tau)\right) \mathrm{d} \tau, & & \text { in } \Omega, \\
\mathbf{u} & =\mathbf{0}, & & \text { on } \partial \Omega .
\end{aligned}\right.
$$

Notice that the homogenized equations (1.21) have exactly the same form as the original ones (1.5) if the original moduli $E_{\infty}(\mathbf{x} / \varepsilon)$ and $G(\mathbf{x} / \varepsilon, t)$ are replaced by the corresponding effective moduli $E_{\infty}^{h o m}$ and $G^{\text {hom }}(t)$. Hence we have replaced a complicated problem, set on a perforated domain, by a classical viscoelastic problem without microstructure.

However, this simplification comes at the cost of having to compute beforehand the values of the homogenized moduli by solving cell problems. In particular, it is necessary to obtain the values of the memory function $G^{\text {hom }}(t)$ for every time $t$. Applying formally the inverse Fourier transform to (1.20) and (1.19), we recover $G^{\text {hom }}(t)$ as

$$
G_{i j k l}^{h o m}(t)= \begin{cases}\int_{\mathcal{Y}_{S}} G_{i j k l}(t)+\left(\int_{\mathcal{Y}_{S}} G(t) e_{\mathbf{y}}\left(\hat{\chi}^{i j}(0)\right)+\sigma^{i j}(t)\right)_{k l}, & \text { for } t>0, \\ 0, & \text { for } t \leq 0,\end{cases}
$$

where the tensor $\sigma^{i j}(t)$ is defined for $t>0$ as a function of some distributions $\phi^{i j}(t), \psi^{i j}(t)$, which are weak solutions of the following quasi-static viscoelastic problem for $t>0$ :

$$
\left\{\begin{array}{rlrl}
-\operatorname{div}_{\mathbf{y}} \sigma^{i j}(t) & =\operatorname{div}_{\mathbf{y}}\left(G(t) e_{\mathbf{y}}\left(\mathbf{p}^{i j}+\widehat{\chi^{i j}}(0)\right)\right), & & \text { in } \mathcal{Y}_{S}, \\
\sigma^{i j}(t) & =-\psi^{i j}(t) \operatorname{Id}+E_{\infty} e_{\mathbf{y}}\left(\phi^{i j}(t)\right)+\int_{0}^{t} G(t-\tau) e_{\mathbf{y}}\left(\partial_{t} \phi^{i j}(\tau)\right) \mathrm{d} \tau, & & \text { in } \mathcal{Y}_{S}, \\
\operatorname{div}_{\mathbf{y}} \phi^{i j} & =0, & & \text { in } \mathcal{Y}_{S}, \\
\sigma^{i j}(t) \mathbf{n} & =-\left(G(t) e_{\mathbf{y}}\left(\mathbf{p}^{i j}+\widehat{\chi^{i j}}(0)\right)\right) \mathbf{n}, & & \text { on } \Gamma, \\
\psi^{i j}, \phi^{i j} \mathcal{Y} \text {-periodic. } &
\end{array}\right.
$$

Note that (1.22) has no initial condition: v.g. if $G$ has a limit for $t \rightarrow 0^{+}$, the problem is elliptic for $t=0$.

Remark 1.8. Proving rigorously the well-posedness of the quasi-static viscoelastic problem (1.22) is difficult in general. More precisely, uniqueness of the solution among distributions can be deduced from the strong ellipticity of (1.19) in the frequency domain. However, this solution may not belong to a functional space such as $\mathrm{L}^{1}\left(\mathbb{R}^{+}, \mathrm{L}^{\infty}(\mathcal{Y}\right.$, Sym $\left.)\right)$. Note that in the particular case where (1.15) is satisfied uniformly in $\omega$ and $G^{\prime}$ belongs to $\mathrm{L}^{1}\left(\mathbb{R}^{+}, \mathrm{L}^{\infty}(\mathcal{Y}\right.$, Sym $\left.)\right),(1.22)$ is well-posed and $\phi^{i j}(t), \psi^{i j}(t)$ belong to $\mathrm{L}_{\text {loc }}^{2}\left(\mathbb{R}^{+}, \mathrm{L}^{\infty}(\mathcal{Y}\right.$, Sym $\left.)\right)$, see [10].

\subsubsection{Memory effects}

An interesting aspect of the homogenization of viscoelastic materials is the appearance of new memory effects caused by the coupling of spatial and temporal variations of the viscoelastic modulus of the material. It has been argued by Tartar [39] that such long-term memory effects are induced by the spatial averaging in the solutions of hyperbolic equations with coefficients oscillating in space. In particular, long-term memory effects have been shown to appear when homogenizing two-phase media coupling an elastic matrix and a Newtonian fluid $[4,12]$, a Voigt body [33], or a Maxwell body [38], even though each component exhibits only instantaneous memory. 
The appearance of new memory effects depend directly on the inhomogeneity of the viscoelastic properties of the material. Indeed, suppose that the dependence on the time and space variables of the material are separable, in the sense that the relaxation function of the wall material is

$$
E(\mathbf{y}, t)=E_{\infty}(\mathbf{y})(1+g(t)) \quad \text { or } \quad G(\mathbf{y}, t)=E_{\infty}(\mathbf{y}) g(t),
$$

where $g$ belongs to $\mathrm{L}^{1}\left(\mathbb{R}_{+}\right)$. Then, thanks to (1.14),

$$
\operatorname{div}_{\mathbf{y}}\left(E_{\infty} e_{\mathbf{y}}\left(\mathbf{p}^{i j}+\widehat{\chi^{i j}}(0)\right)\right)=\nabla \widehat{\eta^{i j}}(0) \quad \text { in } \mathcal{Y}_{S},
$$

and it is immediate from $(1.22)$ that $\sigma^{i j}(t)=-g(t) \widehat{\eta^{i j}}(0)$ for all times. Hence, the effective memory function is

$$
G^{h o m}(t)=\left(E_{\infty}^{h o m}-\frac{|\mathcal{Y}| \gamma p_{a}}{\left|\mathcal{Y}_{S}\right|} \mathrm{Id}\right) g(t) .
$$

As a consequence, when the relaxation function is separable it is enough to solve the local problem only once. The only new memory effect to appear is due to the coupling with the gas in the pores, which increases the elastic bulk modulus but not its viscous counterpart.

On the other hand, if the dependance on space and time is not separable, it is necessary to compute the effective relaxation function for all times or, equivalently, in the Laplace or Fourier transformed domain.

\section{Numerical OFFLine/ONLINE STRATEGy FOR THE GLOBAL DISPERSive PROBLEM}

To further improve our understanding of the mechanics of the lungs, we now consider the numerical solution of the effective equation (1.21). Our objective is to obtain a fast numerical method for the computation of the solutions to the homogenized wave propagation problem (1.21). The strategy we propose consists in two distinct parts:

- Computing the homogenized material parameters, based on a choice of parameters describing the material at the microscopic level. This is a preliminary, offline step, independent of the macroscale geometry or source data, and is described in Section 2.2.

- Integrating in time the propagation of the viscoelastic wave on a given macroscale geometry by an efficient Discontinuous Galerkin method, presented in Sections 2.3 and 2.4.

\subsection{Evaluation of the convolution integral}

Several numerical methods have been developed to solve the initial boundary problem associated with the equations of elasticity or viscoelasticity, with notable applications to propagation of seismic waves or to the determination of the elastic parameters of soft tissue by MRI measurements. The treatment of the convolution term in (1.21) is an important factor in the choice of the method. One specific model and widely used model is the Zener or Standard Linear Solid model, for which the complex modulus is represented with a Debye-type relaxation function:

$$
\widehat{E}(\omega)=E_{0}+\frac{E_{\infty}-E_{0}}{1+i \omega \tau_{0}},
$$

where $E_{\infty}$ is the relaxed elastic modulus, $E_{0}$ is the instantaneous elastic modulus and $\tau_{0}$ is the relaxation time. If $E_{\infty}$ reduces to zero we obtain the popular Maxwell model. The inverse Fourier transform of (2.1) is an exponential function

$$
E(t)= \begin{cases}E_{0}+\left(E_{\infty}-E_{0}\right)\left(1-\exp \left(-\frac{t}{\tau_{0}}\right)\right), & \text { if } t>0, \\ 0, & \text { else, }\end{cases}
$$

and this key characteristic has led to the development of several computationally efficient algorithms for incorporating Standard Linear Solids into Finite Element or Finite Difference codes [36]. The algorithms take the 
form of simple recursion relations, or auxiliary difference equations that are updated along with the standard elastic wave equation, by adding an additional internal variable.

The Zener model is a poor model of viscoelastic behavior for most materials over wide frequency ranges. However because the Fourier transform of most non-Zener models are not exponential functions, they cannot be incorporated directly into Finite Elements or Finite Difference codes using recursion relations or auxiliary difference equations. This is in particular the case of the homogenized model we have obtained. Historically, it was proposed that the integral may be simply discretized by a trapezoidal method. However, this proves too costly in practice since the convolution must be reevaluated at each time step, so the entire history of the deformation must be kept in memory.

An approach used by many investigators (see $v . g$. the review [36]) is to approximate the complex relaxation modulus using a Prony series expansion of the form

$$
E(t) \approx E_{0}-\sum_{i=1}^{n} E_{i}\left(1-\mathrm{e}^{-t / \tau_{i}}\right)=E_{\infty}+\sum_{i=1}^{n} E_{i} \mathrm{e}^{-t / \tau_{p}},
$$

where $n$ is the total number of functions, $E_{i}$ the relaxation modulus and $\tau_{i}$ the relaxation time of the $i$ th function. This model is called the Generalized Maxwell or Wiechert model. Most of the algorithms developed for Zener or Maxwell models can efficiently incorporate multiple relaxation mechanisms as a way to accomodate models with a non-exponential relaxation function. Indeed, in this case, we may transform the convolution integral appearing in the evolution equation (1.21) by introducing additional internal variables to the original set of unkowns. Denoting by $e(t)$ the strain tensor $e(\mathbf{u})$, we have the relation:

$$
\int_{0}^{t} E(t-\tau) \partial_{t} e(\tau) \mathrm{d} \tau=E_{0} e(t)-E_{i} e_{i}
$$

where we have introduced $n$ internal variables $e_{i}$, defined by

$$
e_{i}=\int_{0}^{t} \partial_{t} e(\tau)\left(1-\mathrm{e}^{(\tau-t) / \tau_{i}}\right) \mathrm{d} \tau \quad \text { for } i=1, \ldots, n,
$$

or alternatively satisfying $e_{i}(0)=0$ and the additional ordinary differential equation

$$
\tau_{i} \partial_{t} e_{i}+e_{i}=e .
$$

Hence, the use of the Wiechert model to describe the homogenized material enables us to replace the expensive computation of the convolution, at the cost of introducing additional internal variables.

\subsection{Computation and fitting of the dispersive curve}

Next, we present a method for computing the viscoelastic complex modulus of the homogenized material and its Prony series representation (2.2). This is the first, offline step of the numerical method and can be done ahead of the time-domain computation, with no knowledge of the macroscopic geometry or of the wave data. This computation of the homogenized parameters can be quite expensive, but it has to be done only once for each set of micro-scale parameters.

In this paper, we chose to obtain the coefficients of the Prony series approximating the homogenized relaxation function using the method of nonlinear least-squares. This approach yields good results and can be implemented using standard routines. The approach is to sample the homogenized coefficients and fit the complex modulus with a Prony series by fitting both real and imaginary parts at the same time:

$$
\begin{aligned}
\operatorname{Re}\left(\widehat{E}^{h o m}(\omega)\right) & =E_{\infty}^{h o m}+\sum_{p=1}^{N_{p}} E_{p}\left(\frac{1}{1+\omega^{2} \tau_{p}^{2}}\right), \\
\frac{1}{\omega} \operatorname{Im}\left(\widehat{E}^{h o m}(\omega)\right) & =\operatorname{Re}\left(\widehat{G}^{h o m}(\omega)\right)=\sum_{p=1}^{N_{p}} E_{p}\left(\frac{\tau_{p}}{1+\omega^{2} \tau_{p}^{2}}\right) .
\end{aligned}
$$


Remark 2.1. In [40], it was proposed to fit the modulus in the (real) Laplace transformed domain, but the results were found not to be very stable when comparing the complex moduli in the Fourier frequency domain.

\subsubsection{Solution of the cell problems}

To implement the least-squares method, we sample the complex modulus at a large number of frequencies covering the range of interest. This is achieved by solving the complex elliptic problems (1.14) to compute the frequency-dependent complex correctors $\widehat{\chi^{i j}}(\mathbf{y}, \omega)$. The numerical solution of the cell problems (1.14) is obtained by standard Finite Element methods for a given set of parameters modeling the microscale parenchyma tissue.

Remark 2.2. Note that this step can be quite costly, as it potentially requires solving a large number of independent highly resolved cell problem. However, this work is done offline, and can be trivially parallelelized.

If the cell problems are further parameterized by another parameter, for example by dependence of the coefficients or the cell geometry on the slow variable $\mathbf{x}$, then this computation has also to be done for every point in the macroscopic domain. In this case it would be interesting to consider methods of reduced complexity for solving repeatedly the parameterized cell problems (1.14), v.g. the reduced order basis method as proposed in [5]. The reduced basis method has been applied for such computations in v.g. [25,26].

\subsection{Discontinuous Galerkin discretization}

Assume now that we have a distribution $\left(E_{i}, \tau_{i}\right)$ of relaxation mechanisms describing the viscoelastic complex modulus of the homogenized medium as in (2.2). To solve the problem (1.21), we express the equation system in the first-order velocity-strain formulation. To this end, we introduce the velocity vector $\mathbf{v}=\partial_{t} \mathbf{u}$, and denote the tensorial product of two vectors $\mathbf{a}, \mathbf{b}$ in $\mathbb{R}^{d}$ as $\mathbf{a} \otimes \mathbf{b}$ in $\mathbb{R}^{d \times d}$ with

$$
(\mathbf{a} \otimes \mathbf{b})_{i j}=a_{i} b_{j} .
$$

Then, using the equation of motion (1.21) and the relations (2.3) and (2.4), we formulate the pseudo-conservative system of viscoelastic wave equations in the form of a multi-dimensional conservation law complemented with $n$ relaxation laws on $\Omega$, for $t \geq 0$ :

$$
\left\{\begin{array}{r}
\rho_{S}\left|Y_{S}\right| \partial_{t} \mathbf{v}-\operatorname{div}\left(E_{0}^{h o m} e-E_{i}^{h o m} e_{i}\right)=\mathbf{f}, \\
\partial_{t} e-\operatorname{div}\left(\frac{\mathbf{v} \otimes \mathbf{e}_{j}+\mathbf{e}_{j} \otimes \mathbf{v}}{2}\right)=0, \\
\tau_{1} \partial_{t} e_{1}+e_{1}=e, \\
\vdots \quad \vdots \\
\tau_{n} \partial_{t} e_{n}+e_{n}=e,
\end{array}\right.
$$

with zero initial and boundary conditions.

Note that each additional relaxation mechanism adds a further 3 variables in dimension two and 6 in dimension three, one for each strain component of the additional internal variable. Therefore, the system consisting of 5 or 9 equations in the purely elastic case is complemented by an additional $3 n$ or $6 n$ equations (depending on the dimension) in the viscoelastic case when we use $n$ relaxation mechanisms.

We denote $\mathbf{W}=(\mathbf{v}, e)$ as the vector composed by the velocity and the strain components and $\mathbf{M}=\left(e_{1}, \ldots, e_{n}\right)$ as the vector composed by the internal strain variable components. The system (2.5) can be rewritten in compact form

$$
\left\{\begin{array}{r}
\partial_{t} \mathbf{W}-\operatorname{div}\left(\mathcal{A}_{1} \mathbf{W}-\mathcal{A}_{2} \mathbf{M}\right)=\mathbf{f}, \\
\mathcal{T} \partial_{t} \mathbf{M}+\mathbf{M}=\mathcal{B} \mathbf{W},
\end{array}\right.
$$


where we define, using the block-matrix notation, the matrices

$$
\begin{aligned}
\mathcal{A}_{1}=\left(\rho_{S}\left|Y_{S}\right|\right)^{-1}\left[\begin{array}{cc}
0 & \frac{\mathbf{e}_{i} \otimes \mathbf{e}_{j}+\mathbf{e}_{j} \otimes \mathbf{e}_{i}}{2} \\
E_{0}^{h o m} & 0
\end{array}\right], & \mathcal{A}_{2}=\left(\rho_{S}\left|Y_{S}\right|\right)^{-1}\left[E_{1}^{\text {hom }} \ldots E_{n}^{\text {hom }}\right], \\
\mathcal{T}=\left[\tau_{1} \mathrm{Id} \ldots \tau_{n} \mathrm{Id}\right], & \mathcal{B}=\left[\left[\begin{array}{c}
0 \\
\mathrm{Id}
\end{array}\right] \ldots\left[\begin{array}{c}
0 \\
\mathrm{Id}
\end{array}\right]\right]^{T} .
\end{aligned}
$$

Note that the dimension of the vector $\mathbf{M}$ and of the matrices $\mathcal{A}_{2}, \mathcal{T}$ and $\mathcal{B}$ depends on the chosen number $n$ of the relaxation mechanisms.

We assume that the computational domain $\Omega$ is composed of $K$ non-overlapping elements (v.g. triangles in dimension two, tetrahedra in dimension three)

$$
\Omega=\bigcup_{k=1}^{K} \mathcal{D}_{k}
$$

and that the solution in each subdomain $\mathcal{D}_{k}$ is well approximated by the local polynomial of degree $p$

$$
\begin{aligned}
& \mathbf{W}^{k}(\mathbf{x}, t)=\mathbf{W}^{k}\left(\mathbf{x}_{i}^{k}, t\right) L_{i}^{k}(\mathbf{x})=\mathbf{W}_{i}^{k}(t) L_{i}^{k}(\mathbf{x}), \\
& \mathbf{M}^{k}(\mathbf{x}, t)=\mathbf{M}^{k}\left(\mathbf{x}_{i}^{k}, t\right) L_{i}^{k}(\mathbf{x})=\mathbf{M}_{i}^{k}(t) L_{i}^{k}(\mathbf{x}),
\end{aligned}
$$

where $\mathbf{x}_{i}^{k}$ are the $N$ grid points in the $k$ th element and $L_{i}^{k}(\mathbf{x})$ is the two- or three-dimensional multivariate Lagrange polynomial based on these points (we refer to [16] for a detailed account of the nodal spatial discretization). Note that

$$
N=\frac{(p+1)(p+2)}{2} \text { in } 2 \mathrm{D}, \quad N=\frac{(p+1)(p+2)(p+3)}{6} \text { in 3D. }
$$

The physical flux is approximated locally as

$$
\mathcal{F}^{k}\left(\mathbf{W}^{k}, \mathbf{M}^{k}\right)=\left(\mathcal{A}_{1} \mathbf{W}_{i}^{k}-\mathcal{A}_{2} \mathbf{M}_{i}^{k}\right) L_{i}^{k}(\mathbf{x}) .
$$

We implement a Galerkin projection approach and integrate (2.5) against the $N$ local test functions $L_{k}^{i}(\mathbf{x})$ for each element. After integration by parts, we obtain the semi-discrete form of the scheme

$$
\left\{\begin{aligned}
\int_{\mathcal{D}_{k}}\left(\partial_{t} \mathbf{W}^{k}-\operatorname{div} \mathcal{F}^{k}\right) L_{i}^{k}(\mathbf{x}) & =\int_{\partial \mathcal{D}_{k}}\left(\mathcal{F}^{k}-\mathcal{F}^{*}\right) L_{i}^{k}(\mathbf{x}) \mathbf{n}+\int_{\mathcal{D}_{k}} \mathbf{f}(\mathbf{x}, t) L_{i}^{k}(\mathbf{x}), \\
\mathcal{T} \partial_{t} \mathbf{M}^{k}+\mathbf{M}^{k} & =\mathcal{B} \mathbf{W}^{k}
\end{aligned}\right.
$$

As numerical flux we use the local Lax-Friedrichs flux [22]

$$
\mathcal{F}^{*}=\mathcal{F}^{*}\left(\mathbf{W}^{+}, \mathbf{M}^{+}, \mathbf{W}^{-}, \mathbf{M}^{-}\right)=\mathcal{A}_{1} \frac{\mathbf{W}^{+}+\mathbf{W}^{-}}{2}-\mathcal{A}_{2} \frac{\mathbf{M}^{+}+\mathbf{M}^{-}}{2}-\frac{c}{2}\left(\mathbf{W}^{+}-\mathbf{W}^{-}\right),
$$

where $\mathbf{W}^{-}, \mathbf{M}^{-}$refers to the local solution, $\mathbf{W}^{+}, \mathbf{M}^{+}$refers to the neighboring solutions, and $c$ is the maximum eigenvalue of the flux Jacobian $\mathcal{A}_{1}$, which here is the maximum wave speed in the unrelaxed elastic medium.

To show the stability of this semi-discrete scheme, we note that the additional equation appearing in the system (2.8) does not involve any spatial operator as $\mathcal{T}$ is a diagonal matrix and corresponds to a damping of the solution. Hence the stability of the semi-discrete scheme (2.8) follows from the stability of the semi-discrete scheme for the wave equation when one uses a monotone flux such as the Lax-Friedrichs flux. 


\subsection{Implicit-explicit time-stepping scheme}

We will integrate in time using a high-order Runge-Kutta scheme. It is possible to use a fully explicit time-integration scheme, which is simple to implement and the most efficient for low levels of stiffness, but imposes a stability-based time-step restriction. Considering the scheme (2.8), we note that there are two different mechanisms potentially controlling the maximum possible time-step:

- the usual Courant-Friedrichs-Levy condition, induced by the discretization of a first-order operator and which is controlled by the geometry of the spatial discretization and the wave speed,

- the characteristic times $\tau_{i}$ associated with the relaxation mechanisms, which a priori may be arbitrarily small. Indeed the range of relaxation times can be very wide, even as large as $10^{10}$ for a Maxwell system [36], and a suitable time integrator shoud be both stable and accurate for small and large values of the relaxation time.

To adress this second limitation, we implement an implicit-explicit Runge-Kutta method proposed by Kennedy and Carpenter [19], see also [17]. This allows us to keep the efficiency of the explicit approach and avoid solving the large, non-stiff linear system associated with the wave equation discretized by the first equation in $(2.8)$. At the same time, it integrates implicitly and accurately the system of ODEs formed by the second equation of the problem, which effectively forms a diagonal linear system which is trivial to solve. Let us write the system $(2.8)$ as

$$
\left\{\begin{array}{l}
\frac{\mathrm{d} \mathbf{W}}{\mathrm{d} t}=\mathbf{F}^{e x}(\mathbf{W}, \mathbf{M}, t), \\
\frac{\mathrm{d} \mathbf{M}}{\mathrm{d} t}=\mathbf{F}^{i m}(\mathbf{W}, \mathbf{M}, t) .
\end{array}\right.
$$

Then the time-stepping scheme writes as follows. To compute $\mathbf{W}(t+\Delta t)=\mathbf{W}^{(n+1)}$ with an $s$-stage IMEX-RK method,

$$
\begin{aligned}
& \left\{\begin{array}{l}
\mathbf{W}^{(i)}=\mathbf{W}^{(n)}+\Delta t \sum_{j=1}^{s} a_{i j}^{e x} \mathbf{F}^{e x}\left(\mathbf{W}^{(j)}, \mathbf{M}^{(j)}, t^{(n)}+c_{j} \Delta t\right), \\
\mathbf{M}^{(i)}=\mathbf{M}^{(n)}+\Delta t \sum_{j=1}^{s} a_{i j}^{i m} \mathbf{F}^{i m}\left(\mathbf{W}^{(j)}, \mathbf{M}^{(j)}, t^{(n)}+c_{j} \Delta t\right),
\end{array}\right. \\
& \left\{\begin{array}{l}
\mathbf{W}^{(n+1)}=\mathbf{W}^{(n)}+\Delta t \sum_{j=1}^{s} b_{i} \mathbf{F}^{e x}\left(\mathbf{W}^{(i)}, \mathbf{M}^{(j)}, t^{(n)}+c_{i} \Delta t\right), \\
\mathbf{M}^{(n+1)}=\mathbf{M}^{(n)}+\Delta t \sum_{j=1}^{s} b_{i} \mathbf{F}^{i m}\left(\mathbf{W}^{(i)}, \mathbf{M}^{(j)}, t^{(n)}+c_{i} \Delta t\right),
\end{array}\right.
\end{aligned}
$$

where $\mathbf{W}^{(i)}=\mathbf{W}\left(t^{(n)}+c_{i} \Delta t\right)$, and the fixed scalar coefficients $a_{i j}^{e x}, a_{i j}^{i m}, b_{j}$ and $c_{i}$ determine the accuracy and stability of the given RK scheme.

The matrix $A^{e x}$ is characteristic of a fully explicit Runge-Kutta scheme, usually referred to as an ERK scheme, as it is a strictly lower triangular matrix. The matrix $A^{i m}$ is associated with a singly diagonally implicit Runge-Kutta scheme, or ESDIRK scheme, and is a lower triangular matrix with nonzero identical diagonal coefficients. Note that the two schemes are coupled through the nodes $c_{i}$ and the weights $b_{j}$. The precise values of the coefficients for different IMEX schemes can be found in $v . g$. [19].

Coupling the two parts of the scheme is straightforward: at each stage of the RK computation, the explicit variable $\mathbf{W}$ (displacement and stress) is integrated to obtain $\mathbf{W}^{(i)}$, and then the implicit variable $\mathbf{M}$ (internal variables) are integrated using the explicitly computed stress as a source term. 
TABle 1. Parameter estimates for the fluid-structure model (1.5).

\begin{tabular}{lcc}
\hline Parameter & Description & Estimate \\
\hline$\mu$ & Shear Modulus of the Elastic Fibers & $10^{5} \mathrm{~Pa}$ \\
$\nu$ & Shear viscosity of the Ground Substance & $10^{3} \mathrm{~Pa} \cdot \mathrm{s}$ \\
$\rho_{s}$ & Density of the Alveolar Wall & $10^{3} \mathrm{Kg} \cdot \mathrm{m}^{-3}$ \\
$p_{a}$ & Atmospheric pressure & $10^{5} \mathrm{~Pa}$ \\
\hline
\end{tabular}

\section{Numerical Results}

In this section, we present the results of numerical experiments in a two-dimensional setting. First, we solve the cell problems (1.14) on a few examples of cell geometries and parameters using the Finite Elements software FreeFem $++[15]$. Then, we use the C++ Least-Squares library levmar [24] to fit the computed dispersion curve to a Prony series. Finally, we implement the numerical scheme (2.8) using the Discontinuous Galerkin solver Hedge [20].

\subsection{Effective viscoelastic modulus computation}

To show that the inhomogeneity of the material at the microscale results in interesting effects, we compute the effective viscoelastic complex modulus for a two-dimensional test micro-scale geometry. The chosen periodicity cell is the unit square. Note that this choice has significant effects on the resulting properties of the homogenized material, as will become clear from the wave propagation simulations. To model the connective tissue of the parenchyma, we propose to study a periodic, connected, elastic skeleton presenting inclusions filled with a viscous material, modeling respectively the network of elastic fibers sustaining the lungs and the ground substance and fluids contained in the parenchyma.

Remark 3.1. It is not possible in this two-dimensional setting to model both phases of the material as connected, even though both the elastic fiber network and the blood capillary network are connected in the real lung parenchyma.

In our model, the domain $\mathcal{Y}_{S}$ is divided into two distinct parts, noted $\mathcal{Y}_{S}^{f}$ and $\mathcal{Y}_{S}^{v}$, on which the material's parameters $E_{\infty}(\mathbf{y})$ (relaxed elastic modulus) and $G(\mathbf{y}, t)$ (memory function), defined by (1.6), are constant with respect to the space variable, see Figure 2. We further assume that $\mathcal{Y}_{S}^{f}$ is connected. Assuming that the material is locally isotropic, the mechanical properties of the elastic substance, dominated by the purely elastic reponse, are given by the relations

$$
E_{\infty}=\mu \mathrm{Id} \quad \text { and } \quad G \approx 0
$$

where $\mu$ is the shear modulus of the elastic substance. Several choices are possible for the viscous filling, and we show results for a Kelvin-Voigt body, satisfying the relations

$$
E_{\infty} \approx 0 \quad \text { and } \quad G=\nu \delta(t),
$$

where $\nu$ is an effective viscosity and $\delta$ the Dirac distribution. Note that to satisfy (1.7), $E_{\infty}$ can be taken to be very small in the viscous material but should be definite positive, although this is not a theoretical restriction in this case (see $v . g$. [12] or the analysis of viscoelastic fluids in [10]). We consider physiologically relevant values for the parameters, as discussed in $v . g$. [28]. Let us note that there are very few available studies of the detailed mechanics of the constituents of the alveolar wall, see $v . g$. [31].

Remark 3.2. We consider a domain $\mathcal{Y}_{S}$ symmetric with respect to the axis $O X$ and $O Y$, so it is possible to use only a quarter of the cell as the computational domain. Moreover, the six values in $\widehat{E}^{\text {hom }}$ reduces to three different values in this case. 


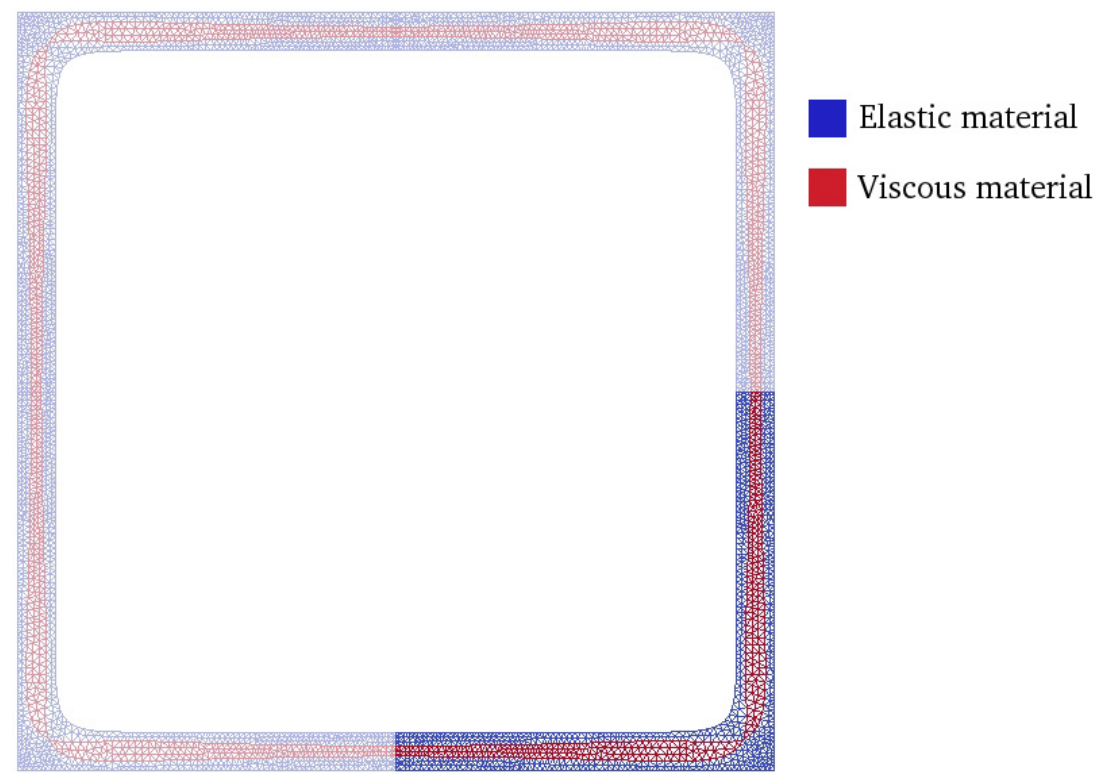

FiguRE 2. Computational mesh representing the test geometry.

Figure 3 shows the results. We observe that the homogenized viscoelastic properties of the material are very different from the behavior of either the elastic, viscous or gas component. In particular, the behavior of pressure waves can be predicted by analyzing the diagonal components of the viscoelastic tensor $\mathcal{G}_{1111}^{\text {hom }}$ and $\mathcal{G}_{1122}^{\text {hom }}$. One observation is that the effective stiffness of the homogenized material goes from the value of the gas compressibility at low frequencies, equal to $\gamma p_{a} \approx 1.4 \times 10^{5} \mathrm{~Pa}$, as predicted by the Rice model [30], to a much higher value for frequencies above $10 \mathrm{kHz}$. This result is consistent with experimental observations [32], although it would be necessary to study numerically more realistic 3D geometries to quantitatively verify this assertion. Hence, it seems that the low-frequency limit is not very dependent on the microstructure, as it depends mainly on the compressibility of the gas content. On the other hand, the high-frequency behavior of the homogenized material is significantly affected by the geometric configuration of the microstructure as well as the relaxation moduli of the various components of the parenchyma. Another observation is that the loss factor, defined as the ratio between the imaginary part and the real part of the compressibility, peaks at a frequency of a few $\mathrm{kHz}$, which indicates a very high attenuation of the pressure waves in the medium in a band of frequencies around this peak. Again, this is consistent with the experimental observation that there is extreme attenuation of sound waves in the audible range through the lungs' parenchyma above $1.5 \mathrm{kHz}$.

\subsection{Fitting the dispersion curve}

The next step consists in the fitting of the curve obtained by a Prony series using the Least-Squares method. We adjust both the coefficients and the relaxation times $\left(E_{p}, \tau_{p}\right)$ in the expansion (2.2) using a nonlinear leastsquares procedure as discussed in [3]. Note that for the stability of the time-domain integration method, the coefficients $\tau_{p}$ and $E_{p}$ should be positive, and this must be incorporated as a constraint in the least-squares algorithm. To show the effect of the number of terms in the Prony series approximation, the number $n$ of relaxation mechanisms is varied by 1,2, 5 and 9 and the best results are shown in Figure 4 for the first coefficient $\mathcal{G}_{1111}^{\text {hom }}$. The choice of the number of relaxation modes is important. We have seen that each additional relaxation mode increases significantly the number of degrees of freedom involved for solving the homogenized problem (1.21) in the time domain and hence the associated memory cost, since we need $5+3 n$ degrees of freedom in $2 \mathrm{D}$ and $9+6 n$ in $3 \mathrm{D}$ for each grid point in the mesh. Hence, it is highly desirable to keep the 

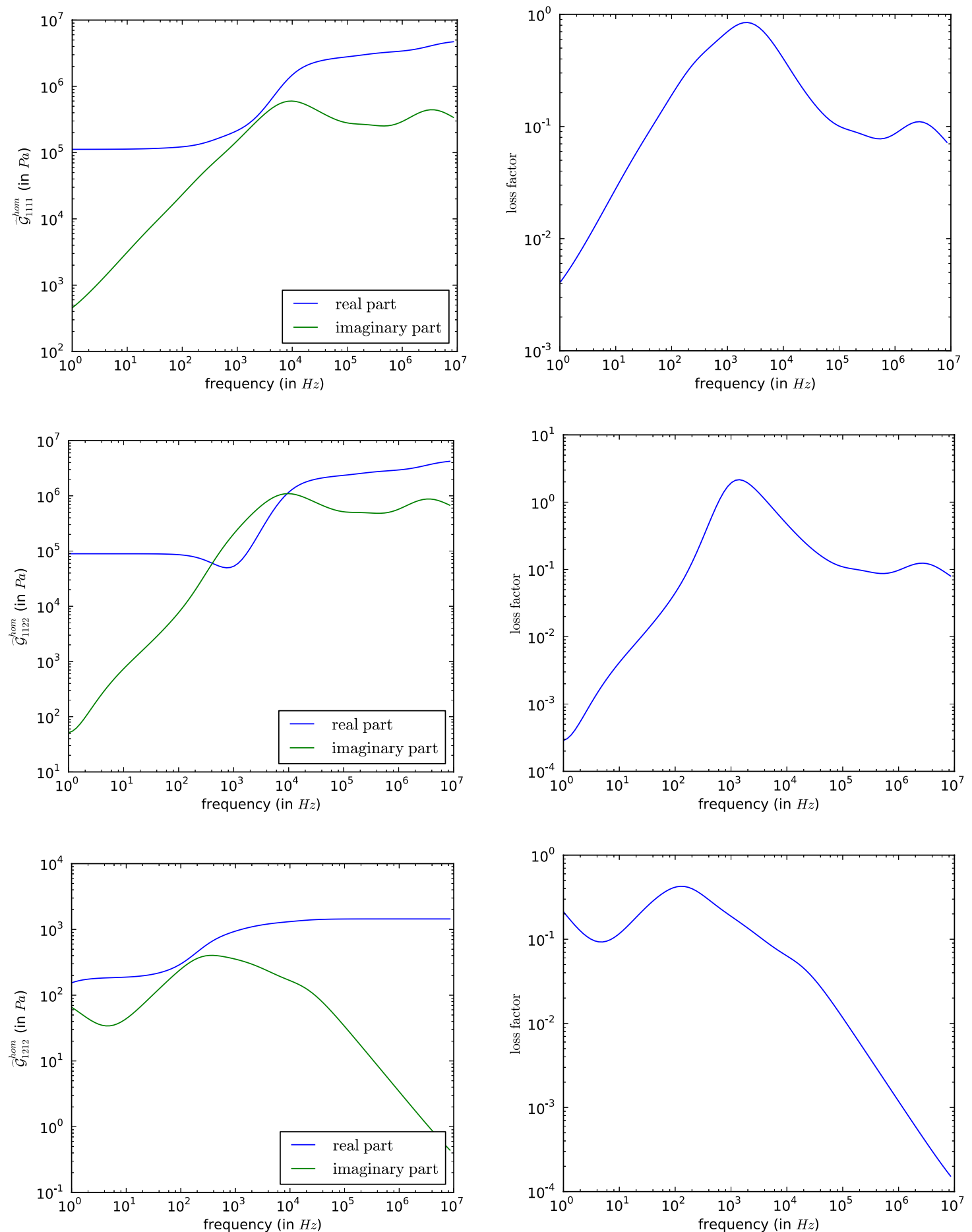

FiguRE 3. The three nonzero complex coefficients of the viscoelastic homogenized tensor (in Pa) as a function of frequency (in $\mathrm{Hz}$ ) obtained by the formula (1.17). On the left we plot the real and imaginary parts, and on the right the loss factor which is the quotient of the imaginary over the real part of the coefficient. 


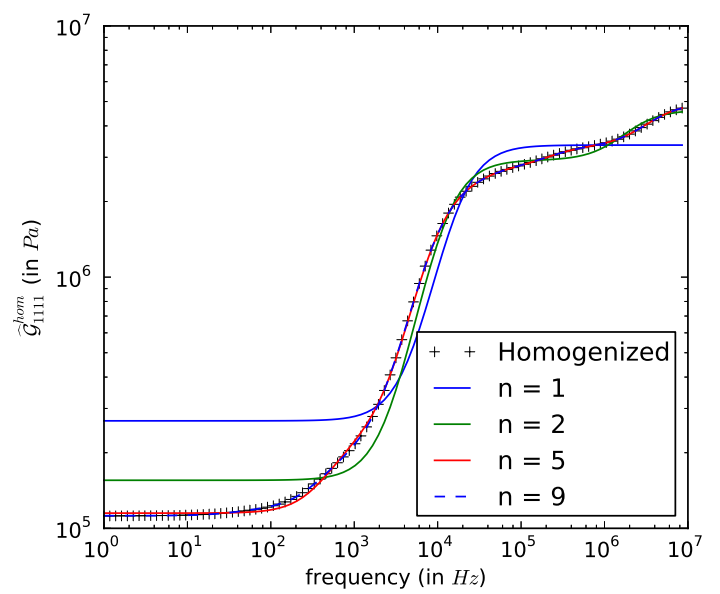

(a) Effective modulus

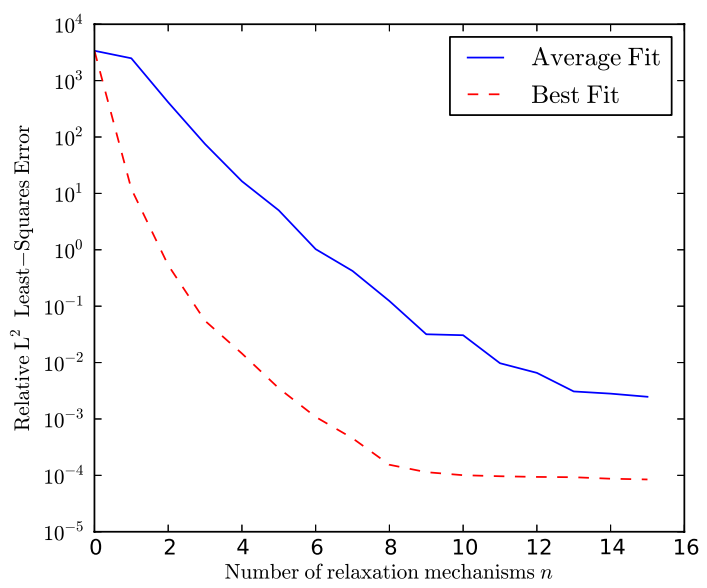

(c) Best and average fit obtained

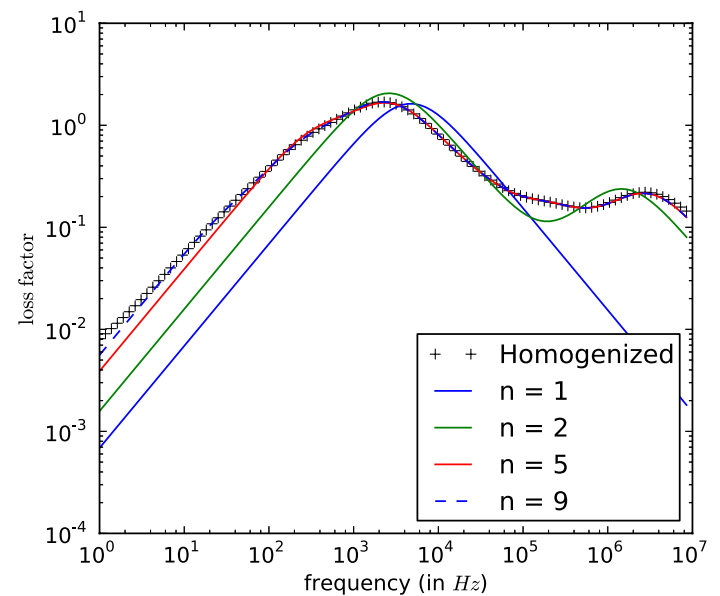

(b) Effective loss factor

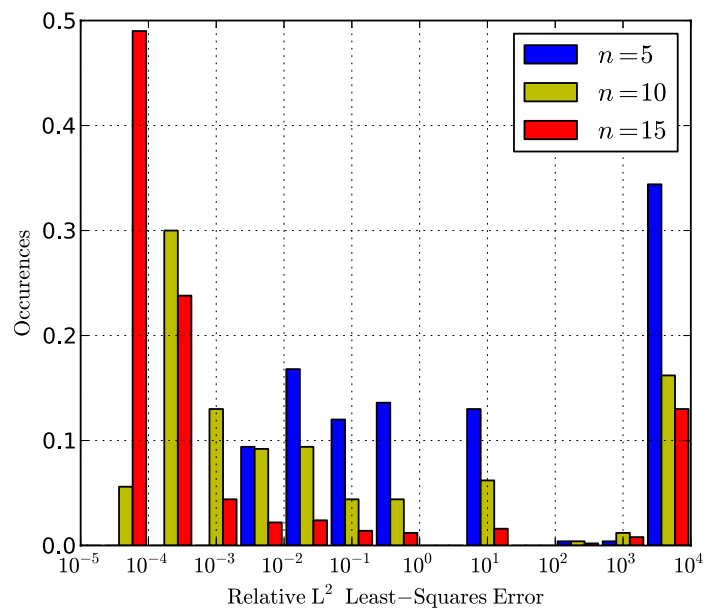

(d) Distribution of the error

FiguRE 4. Effect of the number of fitting terms.

number of relaxation modes as low as possible to ensure both reasonable memory costs and an acceptable error level. To achieve this, it is best not to preset the relaxation times $\tau_{p}$, as proposed in [34], but to optimize their distribution.

The frequency range considered covers 7 orders of magnitude, and we see that 9 relaxation mechanisms are necessary to capture precisely the behavior of the homogenized material. Note that above this number, the residual error levels off. For practical purposes, it may be necessary to reduce the frequency range of the optimization procedure and hence the number of relaxation frequencies to avoid using excessive computer memory.

An important aspect of the method is the choice of the distribution of the set of relaxation times used to initialize the nonlinear least-squares procedure. While a reasonable fit can usually be obtained by uniformly distributing the initial relaxation times, experience shows that the results may be considerably improved by the right choice of initial distribution of relaxation times. Here, the algorithm is simply initialised with a set of relaxation times chosen at random, and the optimisation is repeated until a good fit is found. To show this effect 
TABLE 2. Discretization parameters for the pulse propagation simulations.

\begin{tabular}{lcccc}
\hline Dominant frequency & Disk radius & Timestep & Mesh size & Discretization order \\
\hline $10 \mathrm{~Hz}$ & $10 \mathrm{~m}$ & $120 \mu \mathrm{s}$ & 2500 triangles & 4 \\
$2000 \mathrm{~Hz}$ & $10 \mathrm{~cm}$ & $1.2 \mu \mathrm{s}$ & 2500 triangles & 4 \\
\hline
\end{tabular}

of the initial distribution, we have computed the Least-Squares fit obtained for 500 initial random distributions of relaxation times for values of $n$ between 0 and 15. The results are shown in Figure 4. The error is computed in the $L^{2}$ norm in the frequency range of interest, using a logarithmic change of variables for the frequency. Note that in all cases, some initial distributions of relaxation times lead to extremely bad results. It appears also that the best possible fit can be obtained with $n=9$. Using more relaxation mechanisms makes it easier to find the correct fit but does not increase the precision of the method.

Remark 3.3. It may be interesting, in order to obtain a truly stable and fully automated computational method, to use more advanced fitting techniques. For example, genetic algorithms have been proposed in v.g. [8], as well as hybrid particle swarm-least squares optimization in [18], for the similar problem of fitting the frequency-dependent permittivity of a dielectric material to a Debye series.

\subsection{Wave propagation computations}

Finally, we have implemented the Discontinuous Galerkin numerical scheme for the time-dependent homogenized problem (1.21) described in Sections 2.3 and 2.4. To probe the frequency-dependent attenuation of the homogenized material, we have computed the propagation of a two-dimensional pulse in the disk-shaped domain at various frequencies. We introduce a small volumic source at the center of the disk by setting $\mathbf{f}=s(t) \mathrm{e}^{-|\mathbf{x}|^{2} / \delta^{2}} \mathbf{x}$, where $\delta$ is a small parameter and $s(t)$ is a Ricker function given by $s(t)=\left(1-\left(\omega_{0} t-\beta\right)^{2}\right) \exp \left(\left(\omega_{0} t-\beta\right)^{2}\right)$ with $\beta=3$ as a phase delay parameter and $\omega_{0}$ the dominant frequency of the pulse. Initial conditions for the system are $\mathbf{v}=\mathbf{0}$ and $e, e_{1}, \ldots, e_{n}=0$. We compare the behavior of the viscoelastic medium obtained by homogenization, and approximated by the Prony series, to that of a purely elastic medium presenting the same instantaneous elastic coefficients at the chosen frequency, using the parameters presented in Table 2.

Figure 5 shows three wavefield snapshots at increasing times of the simulation for $\omega_{0}=10 \mathrm{~Hz}$, which corresponds to a low-frequency regime. There is a slight decrease in amplitude in the viscoelastic medium compared to the purely elastic medium, due to absorption, but the signal is shown to propagate without significant distortion.

Figure 6 also shows three snapshots at increasing times of the simulation for $\omega_{0}=2000 \mathrm{~Hz}$, which corresponds to a mid-frequency regime. Now there is a significant difference in the wave propagation in the homogenized medium compared to the reference elastic medium. In the former, the higher frequency content of the pulse is dissipated almost immediately, leading to a drop in amplitude of the signal by a factor of 200 after just a few wavelengths. In this range of frequencies, the homogenized medium does not propagate the signal which is absorbed extremely quickly. We note also that the behavior is markedly anisotropic in both the viscoelastic and elastic media.

\subsection{Orthotropic and isotropic behavior}

An important observation is that the homogenized behavior is highly anisotropic at higher frequencies, with waves propagating almost exclusively along the axis $O X$ and $O Y$. This results from the symmetry properties of the square periodicity cell, which ensure that the homogenized material is orthotropic, but not necessarily invariant with respect to rotations. Hence, this is an artefact of the periodicity we have imposed on the material, whereas the real parenchyma does not have preferred directions for sound propagation.

To recover a more realistic isotropic behavior, several options are possible. In $2 \mathrm{D}$, we can recover isotropic behavior by using a hexagonal periodicity cell instead of a square cell. However, this results from a very particular 


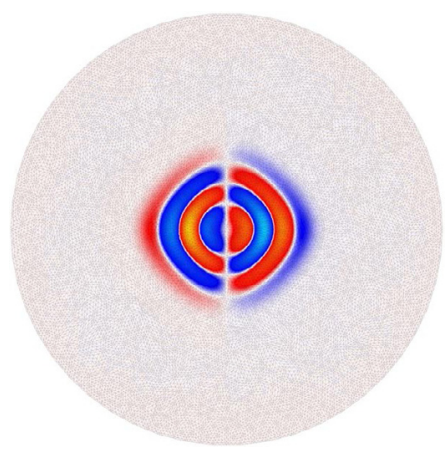

$t=.15 \mathrm{~s}$

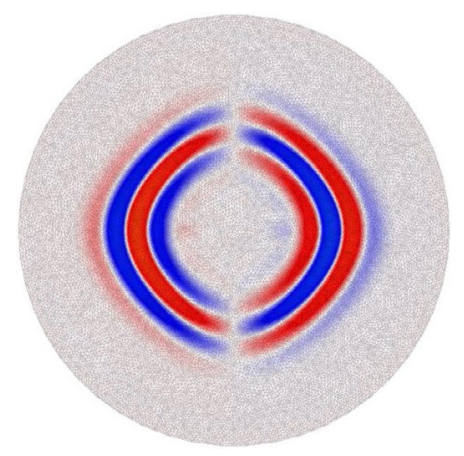

$t=.28 \mathrm{~s}$

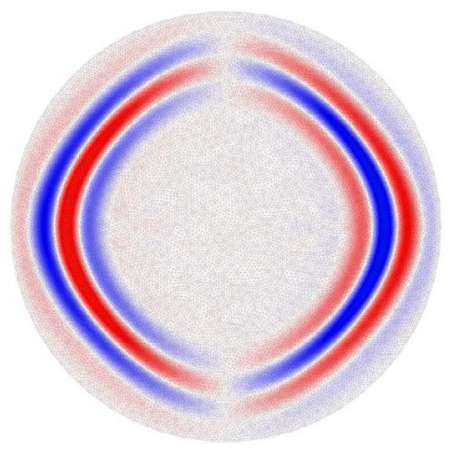

$t=.4 \mathrm{~s}$

(A) Propagation in the viscoelastic homogenized medium with coefficients as in Figure 3.

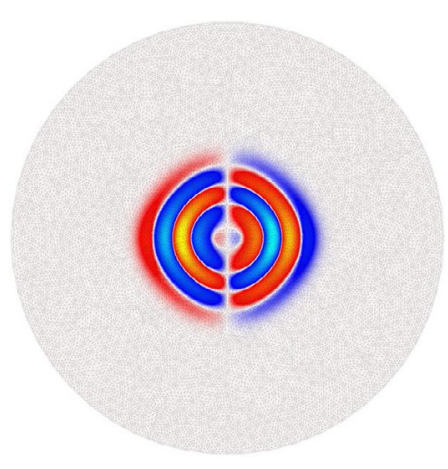

$t=.15 \mathrm{~s}$

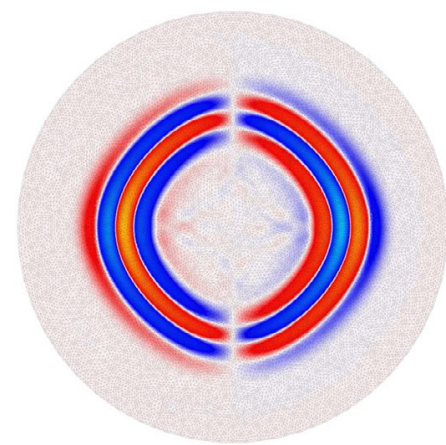

$t=.28 \mathrm{~s}$

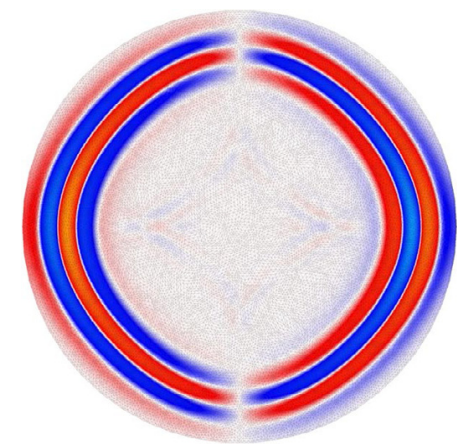

$t=.4 \mathrm{~s}$

(в) Propagation in a purely elastic reference medium with the same elastic coefficients at frequency $10 \mathrm{~Hz}$.

FIGURE 5. Instantaneous snapshots of the propagation of a wave of frequency $10 \mathrm{~Hz}$ as described in Section 3.3. The plots present the first component of the velocity at three different instants. The same color scale is used for all six snapshots.

choice of microstructure model, and there is no equivalent result in 3D. In [28], the homogenized coefficients were averaged directly under an assumption of macroscopic isotropy of the homogenized material, while in [35], the authors used experimental values of Young's modulus and Poisson's ratio for the elastic part of their homogenized parenchyma model.

We propose here another approach which does not involve an arbitrary modification of the homogenized parameters prior to the numerical simulations. The idea is to introduce random rotations inside each element of the discretization, mimicking the random arrangement of the alveoli in the real tissue. This has the effect of a global numerical averaging of the preferred directions of the homogenized material, as the preferred axis of wave propagation now have a random orientation inside each element. To achieve this, we compute a random rotation matrix $R^{k}$ for each element $\mathcal{D}_{k}$ of the discretization. The relaxation moduli $E_{i}$, which are fourth-order tensors, are then replaced inside each element with the rotated moduli $E_{i}^{k}=R^{k} E_{i}\left(R^{k}\right)^{T}$.

Some numerical results are shown in Figure 7. Although a significant scattering of the wavefield is induced by the randomness of the media, we observe that the behavior is now isotropic, and the signal propagates with no preferred direction. Moreover, note that the artificial randomness does not induce much more dissipation than in the reference homogenized case. 


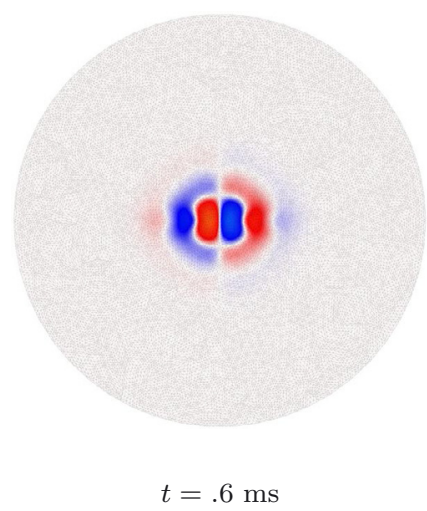

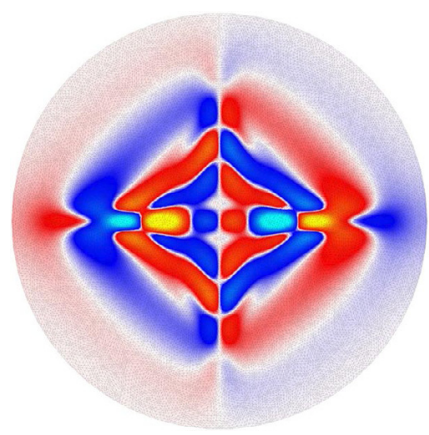

color scale upscaled by 200

$$
t=1.4 \mathrm{~ms}
$$

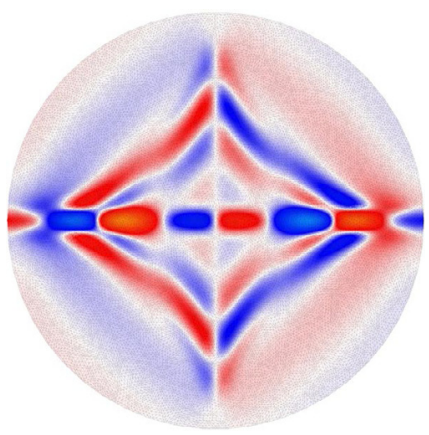

color scale upscaled by 200 $t=2 \mathrm{~ms}$

(a) Propagation in the viscoelastic homogenized medium with coefficients as in Figure 3.

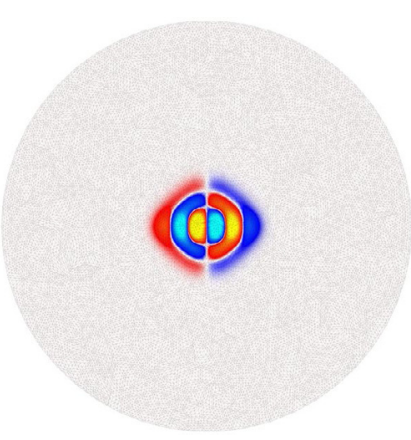

$t=.6 \mathrm{~ms}$

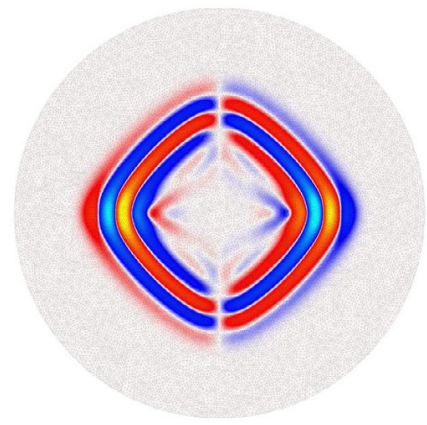

$t=1.4 \mathrm{~ms}$

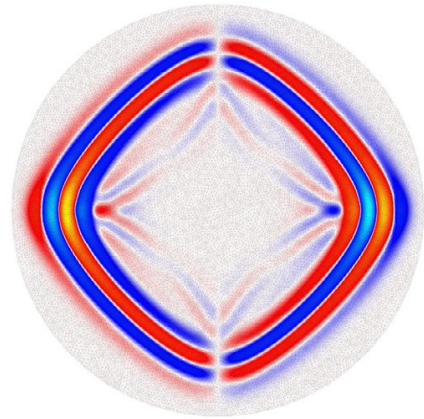

$t=2 \mathrm{~ms}$

(b) Propagation in a purely elastic reference medium with the same elastic coefficients at $2000 \mathrm{~Hz}$.

FiguRE 6. Instantaneous snapshots of the propagation of a wave of frequency $2000 \mathrm{~Hz}$ as described in Section 3.3. The plots present the first component of the velocity at three different instants. In the two last frames of the first row, the color scale for the velocity has been upscaled by a factor of 200 .

\section{Conclusion}

We have presented a homogenized model for viscoelastic porous media such as the lung. This model has enabled us to investigate theoretically and numerically some macroscopic properties of the tissue resulting from the alveolar structure at the microscopic level. The resulting homogenized material has a viscoelastic behavior with new memory effects which have been discussed in detail. Numerical results in 2D show that the material has a band-pass filter behavior. This is in line with recent physiological observations [32], which is interesting given the serious simplifications our model is based on.

Subsequently, a new Discontinuous Galerkin method was developed to deal with the specific numerical issues associated with the presence of the memory effects. To effectively compute the memory effects associated with the viscoelastic nature of the homogenized material, we approximate the memory kernel by a Prony series with multiple relaxation mechanisms using a distribution of relaxation times. The nature of the additional relaxation equations allows us to integrate in time using an implicit-explicit, high-order Runge-Kutta method, keeping the efficiency of the explicit approach allowed by the DG scheme without having to choose a timestep based on the arbitrary small relaxation times. 


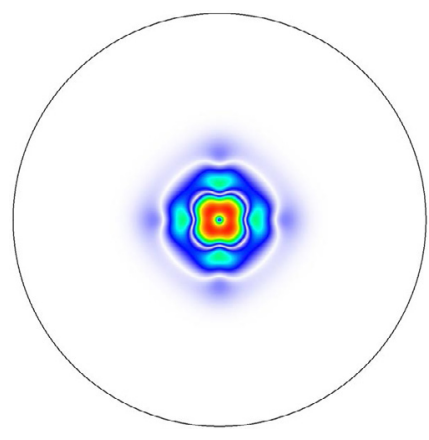

$t=.6 \mathrm{~ms}$

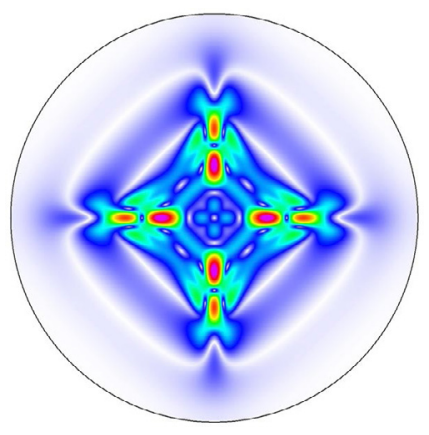

color scale upscaled by 50 $t=1.4 \mathrm{~ms}$

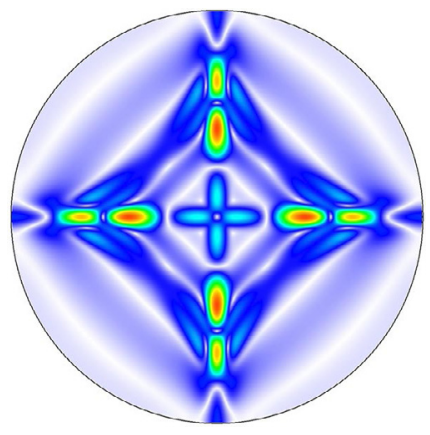

color scale upscaled by 100 $t=2 \mathrm{~ms}$

(a) Propagation in the anisotropic homogenized medium.

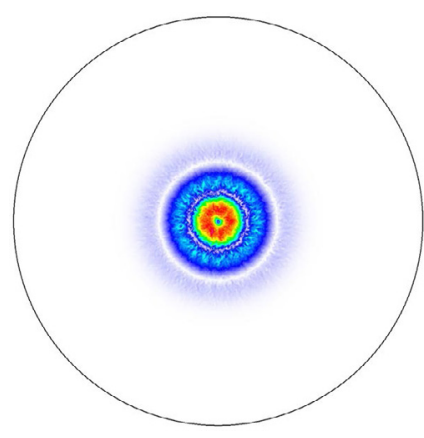

$t=.6 \mathrm{~ms}$

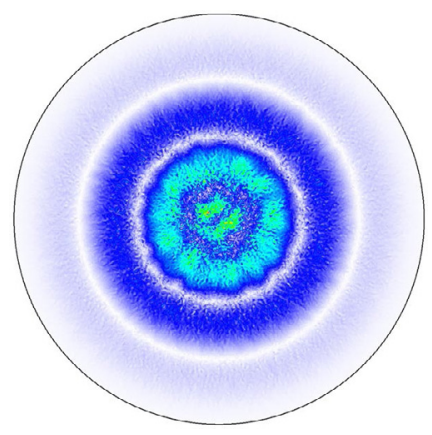

color scale upscaled by 50 $t=1.4 \mathrm{~ms}$

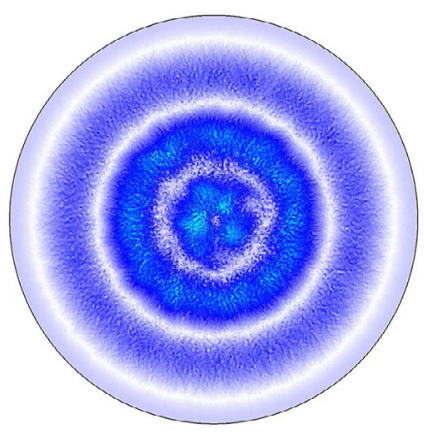

color scale upscaled by 100 $t=2 \mathrm{~ms}$

(b) Propagation in the same homogenized medium with random local rotations of the reference frame.

FiguRE 7. Instantaneous snapshots of the propagation of a wave of frequency $2000 \mathrm{~Hz}$ with and without local random rotations of the reference frame as described in Section 3.3. The plots present the magnitude of the velocity at three different instants. The color scale for the velocity has been upscaled by a factor of 50 and 100 respectively in the two frames to the right.

\section{Appendix A. Proof of Proposition 1.1}

In this Appendix, we give an idea of the proof of the well-posedness of the microscopic problem, Proposition 1.1.

Proof. The proof of this proposition employs classic tools, presented in [23] for example. Let us sketch the main steps. First, we obtain $\mathbf{u}_{\varepsilon}$ as the solution of an auxiliary weak formulation without the pressure, obtained by taking divergence-free test functions and following Galerkin's method:

- choose an orthonormal basis of $\mathbf{V}_{\varepsilon}^{i n c}=\left\{\mathbf{v}_{\varepsilon} \in \mathbf{V}_{\varepsilon}\right.$ : div $\mathbf{v}_{\varepsilon}=0$ a.e. in $\left.\Omega_{\varepsilon}\right\}$, and define finite-dimensional Galerkin spaces of approximation;

- solve the ODE initial value problems obtained by reduction of the auxiliary weak formulation to the finitedimensional Galerkin spaces, in which existence and uniqueness hold;

- bound the sequence of Galerkin approximations using energy estimates to obtain their weak convergence;

- pass to the weak limit and prove that it satisfies the variational formulation (1.9) for any divergence-free test function;

- prove uniqueness by studying the difference between two solutions in the weak formulation. 
In the end, we obtain $\mathbf{u}_{\varepsilon} \in \mathrm{L}^{\infty}\left(0, T ; \mathbf{V}_{\varepsilon}^{\text {inc }}\right) \cap \mathrm{W}^{1, \infty}\left(0, T ; \mathbf{X}_{\varepsilon}\right)$ as solution of the auxiliary variational formulation which reflects (1.9) with only divergence-free test functions. To proceed in the homogenization process, it is crucial to obtain a priori estimates with constants that do not depend on $\Omega_{\varepsilon}$. Taking formally $\partial_{t} \mathbf{u}_{\varepsilon}$ as a test function in (1.9) (this can be rigorously justified by passing to the limit with the Galerkin approximations), we obtain the following energy estimates:

$$
\left\{\begin{aligned}
\frac{1}{2} \frac{\mathrm{d}}{\mathrm{d} t}\left(\int_{\Omega_{\varepsilon}} \rho_{s} \partial_{t} \mathbf{u}_{\varepsilon} \cdot \partial_{t} \mathbf{u}_{\varepsilon}\right. & +\int_{\Omega} E_{\infty}(\mathbf{x} / \varepsilon) e\left(\mathbf{u}_{\varepsilon}\right): e\left(\mathbf{u}_{\varepsilon}\right) \\
& \left.+\frac{\gamma p_{a}}{d\left|\mathcal{Y}_{F}\right| \varepsilon^{d}} \sum_{\mathbf{k} \in \mathbf{Z}_{\varepsilon}^{\Omega}}\left(\int_{\partial \mathcal{B}_{\varepsilon}^{\mathbf{k}}} \mathbf{u}_{\varepsilon} \cdot \mathbf{n}_{\varepsilon}\right)\left(\int_{\partial \mathcal{B}_{\varepsilon}^{\mathbf{k}}} \mathbf{u}_{\varepsilon} \cdot \mathbf{n}_{\varepsilon}\right)\right) \\
& +\int_{\Omega_{\varepsilon}}\left(\int_{0}^{t} G(\mathbf{x} / \varepsilon, t-\tau) e\left(\partial_{t} \mathbf{u}_{\varepsilon}(\tau)\right) \mathrm{d} \tau\right): e\left(\partial_{t} \mathbf{u}_{\varepsilon}\right)=\int_{\Omega_{\varepsilon}} \mathbf{f} \cdot \partial_{t} \mathbf{u}_{\varepsilon} .
\end{aligned}\right.
$$

We integrate in time and use Young's inequality and the coerciveness and dissipativity conditions (1.7), (1.8). Since the energy of the domain is zero at the initial time, we obtain a.e. $t \in[0, T]$ :

$$
\rho_{s}\left\|\partial_{t} \mathbf{u}_{\varepsilon}(t)\right\|_{\mathbf{X}_{\varepsilon}}^{2}+\alpha\left\|e\left(\mathbf{u}_{\varepsilon}(t)\right)\right\|_{L^{2}\left(\Omega_{\varepsilon}\right)}^{2} \leq \int_{0}^{t}\left(\|\mathbf{f}\|_{\mathbf{X}_{\varepsilon}}^{2}+\left\|\partial_{t} \mathbf{u}_{\varepsilon}\right\|_{\mathbf{X}_{\varepsilon}}^{2}\right)
$$

It is well-known that in perforated domains such as $\Omega_{\varepsilon}$, the Korn and Poincar inequality holds with a constant independent of $\varepsilon$ (see for example [2,12]): there exist constants $K$ and $\gamma$, depending only on $\Omega$ and $\mathcal{Y}_{S}$, such that for all $\mathbf{v}_{\varepsilon} \in \mathbf{V}_{\varepsilon}$, we have

$$
\left\|\nabla \mathbf{u}_{\varepsilon}\right\|_{\mathrm{L}^{2}\left(\Omega_{\varepsilon}\right)} \leq K\left\|e\left(\mathbf{u}_{\varepsilon}\right)\right\|_{\mathrm{L}^{2}\left(\Omega_{\varepsilon}\right)}, \quad \quad\left\|\mathbf{u}_{\varepsilon}\right\|_{\mathbf{v}_{\varepsilon}} \leq \gamma\left\|\nabla \mathbf{u}_{\varepsilon}\right\|_{\mathrm{L}^{2}\left(\Omega_{\varepsilon}\right)} .
$$

Thus Gromwall's inequality (in the integral form) yields the estimate,

$$
\left\|\partial_{t} \mathbf{u}_{\varepsilon}(t)\right\|_{\mathbf{X}_{\varepsilon}}^{2}+\left\|\mathbf{u}_{\varepsilon}(t)\right\|_{\mathbf{V}_{\varepsilon}}^{2} \leq C \int_{0}^{T}\|\mathbf{f}\|_{\mathbf{X}_{\varepsilon}}^{2}, \quad \text { a.e. } t \in[0, T]
$$

where $C$ does not depend on $\varepsilon$. Finally, existence, uniqueness and an a priori estimate for the pressure $p_{\varepsilon}$ are obtained from (1.9), following the Proof of Theorem 1 in [4].

Acknowledgements. The authors wish to thank the reviewers for their helpful comments.

\section{REFERENCES}

[1] G. Allaire, Homogenization and two-scale convergence. SIAM J. Math. Anal. 23 (1992) 1482-1518.

[2] L. Baffico, C. Grandmont, Y. Maday and A. Osses, Homogenization of elastic media with gaseous inclusions. Multiscale Model. Simul. 7 (2008) 432-465.

[3] M. Baumgaertel and H.H. Winter, Determination of discrete relaxation and retardation time spectra from dynamic mechanical data. Rheologica Acta 28 (1989) 511-519.

[4] A. Blasselle and G. Griso, Mechanical modeling of the skin. Asymptotic Analysis 74 (2011) 167-198.

[5] S. Boyaval, Reduced-basis approach for homogenization beyond the periodic setting. Multiscale Model. Simul. 7 (2008) 466-494.

[6] R. Burridge and J. Keller, Biot's poroelasticity equations by homogenization, in Macroscopic Properties of Disordered Media, vol. 154 of Lecture Notes in Physics. Springer (1982) 51-57.

[7] J.P. Butler, J.L. Lehr and J.M. Drazen, Longitudinal elastic wave propagation in pulmonary parenchyma. J. Appl. Phys. 62 (1987) 1349-1355.

[8] J. Clegg and M.P. Robinson, A genetic algorithm used to fit Debye functions to the dielectric properties of tissues. 2010 IEEE Congress on Evolutionary Computation (CEC) (2010) 1-8.

[9] F. Dunn, Attenuation and speed of ultrasound in lung: Dependence upon frequency and inflation. J. Acoust. Soc. Am. 80 (1986) 1248-1250. 
[10] M. Fabrizio and A. Morro, Mathematical problems in linear viscoelasticity, vol. 12 of SIAM Studies in Applied Mathematics. SIAM, Philadelphia, PA (1992).

[11] M. Fang, R.P. Gilbert and X. Xie, Deriving the effective ultrasound equations for soft tissue interrogation. Comput. Math. Appl. 49 (2005) 1069-1080.

[12] R.P. Gilbert and A. Mikelić, Homogenizing the acoustic properties of the seabed. I. Nonlinear Anal. 40 (2000) $185-212$.

[13] Q. Grimal, A. Watzky and S. Naili, A one-dimensional model for the propagation of transient pressure waves through the lung. J. Biomech. 35 (2002) 1081-1089.

[14] A. Hanygan, Viscous dissipation and completely monotonic relaxation moduli. Rheologica Acta 44 (2005) 614-621.

[15] F. Hecht, FreeFem + manual (2012).

[16] J.S. Hesthaven and T. Warburton, Nodal discontinuous Galerkin methods, vol. 54 of Texts in Applied Mathematics. Springer, New York (2008).

[17] A. Kanevsky, M.H. Carpenter, D. Gottlieb and J.S. Hesthaven, Application of implicit-explicit high order Runge-Kutta methods to discontinuous-Galerkin schemes. J. Comput. Phys. 225 (2007) 1753-1781.

[18] D.F. Kelley, T.J. Destan and R.J. Luebbers, Debye function expansions of complex permittivity using a hybrid particle swarmleast squares optimization approach. Antennas Propagation IEEE Trans. 55 (2007) 1999-2005.

[19] C.A. Kennedy and M.H. Carpenter, Additive Runge-Kutta schemes for convection-diffusion-reaction equations. Appl. Numer. Math. 44 (2003) 139-181.

[20] A. Kloeckner, Hedge: Hybrid and Easy Discontinuous Galerkin Environment. http://www.cims.nyu.edu/ kloeckner/ (2010).

[21] S.S. Kraman, Speed of low-frequency sound through lungs of normal men. J. Appl. Phys. (1983) 1862-1867.

[22] R.J. LeVeque, Numerical methods for conservation laws. Lectures in Mathematics ETH Zürich. Birkhäuser, Basel (1990).

[23] J.-L. Lions and E. Magenes, Problèmes aux limites non homogènes et applications, vol. 1 of Travaux et Recherches Mathématiques. Dunod, Paris (1968).

[24] M. Lourakis, levmar: Levenberg-Marquardt nonlinear least squares algorithms in C/C++. http://www.ics.forth.gr/ 〜ourakis/levmar/ (2004).

[25] Y. Maday, N. Morcos and T. Sayah, Reduced basis numerical homogenization for scalar elliptic equations with random coefficients: application to blood micro-circulation. Submitted to SIAM J. Appl Math. (2012).

[26] N. Morcos, Modélisation mathématique et simulation de systèmes microvasculaires. Ph.D. thesis, Université Pierre et Marie Curie (2011).

[27] G. Nguetseng, A general convergence result for a functional related to the theory of homogenization. SIAM J. Math. Anal. 20 (1989) 608-623.

[28] M.R. Owen and M.A. Lewis, The mechanics of lung tissue under high-frequency ventilation. SIAM J. Appl. Math. 61 (2001) 1731-1761.

[29] H. Pasterkamp, S.S. Kraman and G.R. Wodicka, Respiratory sounds. advances beyond the stethoscope. Am. J. Respir. Crit. Care Med. 156 (1997) 974.

[30] D.A. Rice, Sound speed in pulmonary parenchyma. J. Appl. Physiol. 54 (1983) 304-308.

[31] E. Roan and M.W. Waters, What do we know about mechanical strain in lung alveoli? Am. J. Physiol. Lung Cell Mol. Physiol. 301 (2011) 625-635.

[32] D. Rueter, H.P. Hauber, D. Droeman, P. Zabel and S. Uhlig, Low-frequency ultrasound permeates the human thorax and lung: a novel approach to non-invasive monitoring. Ultraschall Med. 31 (2010) 53-62.

[33] E. Sanchez-Palencia, Vibration of mixtures of solids and fluids, in Non-Homogeneous Media and Vibration Theory, vol. 127 of Lecture Notes in Physics. Springer (1980) 158-190.

[34] R.A. Schapery, A simple collocation method for fitting viscoelastic models to experimental data. GALCIT SM 63 (1961) 23.

[35] M. Siklosi, O.E. Jensen, R.H. Tew and A. Logg. Multiscale modeling of the acoustic properties of lung parenchyma. ESAIM: Proc. 23 (2008) 78-97.

[36] J. Sorvari and J. Hämäläinen, Time integration in linear viscoelasticity - a comparative study. Mech. Time-Dependent Mater. 14 (2010) 307-328

[37] B. Suki, S. Ito, D. Stamenović, K.R. Lutchen and E.P. Ingenito, Biomechanics of the lung parenchyma: critical roles of collagen and mechanical forces. J. Appl. Physiol. 98 (2005) 1892-1899.

[38] P. Suquet, Linear problems. In Homogenization Techniques for Composite Media, vol. 272 of Lecture Notes in Physics. Edited by Enrique Sanchez-Palencia and André Zaoui. Springer (1987) 209-230.

[39] L. Tartar, The general theory of homogenization. A personalized introduction, vol. 7 of Lecture Notes of the Unione Matematica Italiana. Springer (2009).

[40] Y.-M. Yi, S.-H. Park and S.-K. Youn, Asymptotic homogenization of viscoelastic composites with periodic microstructures. Int. J. Solids Struct. 35 (1998) 2039-2055. 